OPEN ACCESS

Edited by:

Peter Wyper,

Durham University, United Kingdom

Reviewed by:

Sanjiv K. Tiwari,

Lockheed Martin Solar and Astrophysics Laboratory (LMSAL),

United States

Jiansen $\mathrm{He}$

Peking University, China Judy Karpen,

NASA GSFC, United States

${ }^{*}$ Correspondence:

Brigitte Schmieder

brigitte.schmieder@obspm.fr, schmieder.brigitte@kuleuven.be

Specialty section:

This article was submitted to

Stellar and Solar Physics,

a section of the journal

Frontiers in Astronomy and Space

Sciences

Received: 22 November 2021

Accepted: 21 January 2022

Published: 14 February 2022

Citation:

Schmieder B (2022) Solar Jets: SDO

and IRIS Observations in the

Perspective of New MHD Simulations.

Front. Astron. Space Sci. 9:820183.

doi: $10.3389 /$ fspas.2022.820183

\section{Solar Jets: SDO and IRIS Observations in the Perspective of New MHD Simulations}

\author{
Brigitte Schmieder ${ }^{1,2,3 *}$
}

${ }^{1}$ LESIA, Observatoire de Paris, Université PSL, CNRS, Sorbonne Université, Université de Paris, 5 Place Jules Janssen, Meudon, France, ${ }^{2}$ Dept. Mathematics, Centre for mathematical Plasma Astrophysics, KU Leuven, Leuven, Belgium, ${ }^{3}$ School of Physics and Astronomy, University of Glasgow, Glasgow, United Kingdom

Solar jets are observed as collimated plasma beams over a large range of temperatures and wavelengths. They have been observed in $\mathrm{H}$ a and optical lines for more than 50 years and called surges. The term "jet" comes from X-ray observations after the launch of the Yohkoh satellite in 1991. They are the means of transporting energy through the heliosphere and participate to the corona heating and the acceleration of solar wind. Several characteristics have been derived about their velocities, their rates of occurrence, and their relationship with CMEs. However, the initiation mechanism of jets, e.g. emerging flux, flux cancellation, or twist, is still debated. In the last decade coordinated observations of the Interface Region Imaging Spectrograph (IRIS) with the instruments on board the Solar Dynamic Observatory (SDO) allow to make a step forward for understanding the trigger of jets and the relationship between hot jets and cool surges. We observe at the same time the development of 2D and 3D MHD numerical simulations to interpret the results. This paper summarizes recent studies of jets showing the loci of magnetic reconnection in null points or in bald patch regions forming a current sheet. In the pre-jet phase a twist is frequently detected by the existence of a mini filament close to the dome of emerging flux. The twist can also be transferred to the jet from a flux rope in the vicinity of the reconnection by slippage of the polarities. Bidirectional flows are detected at the reconnection sites. We show the role of magnetic currents detected in the footprints of flux rope and quasi-separatrix layers for initiating the jets. We select a few studies and show that with the same observations, different interpretations are possible based on different approaches e.g. non linear force free field extrapolation or 3D MHD simulation.

Keywords: solar jet, solar surge, solar flare, magnetic reconnection, EUV spectroscopy

\section{HISTORICAL STUDIES}

Solar jets are transient phenomena considered being means of energy and mass transport in the solar atmosphere. These are observed in multiple temperatures and wavelengths from $\mathrm{H} \alpha$ (for more than 50 years) to X-rays after the launch of the Yohkoh satellite in August 1991. Their kinematic characteristics (velocity, acceleration, and recurrence) have been derived using different space-borne satellites and ground-based observatories [see recent reviews of Innes et al. (2016); Raouafi et al. (2016); Hinode Review Team (2019); Shen (2021); De Pontieu et al. (2021); Schmieder et al. (2022)]. 
Before describing the present day state of the art of jets, let us look at how surge and jet topic develops through historical studies leading to our present knowledge. The development of instruments with higher and higher spatial and temporal resolution certainly helps us make a step forward in our knowledge so that the cartoons proposed in the late 90's become magnetohydrodynamic (MHD) numerical simulations in three dimensions (3D).

\subsection{Spectroscopic Analysis}

Mass ejections such as sprays, eruptive prominences, and surges have often been observed in $\mathrm{H} \alpha$ and other visible lines (Roy, 1973; Tandberg-Hanssen, 1974). Most of the results deduced from spectroheliograms or filtergrams concern the projected trajectories of the material on the disk and the determination of velocity and acceleration.

In the 1980's, new surge observations were obtained using spectroscopy from space and ground instruments. This is how the first Dopplergrams of jets were obtained with the Ultraviolet Spectro-Polarimeter (UVSP) instrument on board the Solar Maximum Mission in 1980 Woodgate et al. (1980), and consequently new observational results appear at that time.

Coordinated UVSP observations with the Multi-Subtractive Double Pass (MSDP) spectrograph operating on the solar tower of Meudon (Mein, 1977) allowed to obtain full Dopplermaps $(1 \mathrm{~min} \times 8 \mathrm{~min})$ in $\mathrm{H} \alpha$ and in $1,548 \AA \mathrm{C}$ IV lines with the UVSP with a cadence of $30 \mathrm{~s}$ and a spatial resolution of three $\operatorname{arcsec}$ in $\mathrm{C}$ IV and 1 arcsec in $\mathrm{H} \alpha$, respectively. Surges in $\mathrm{H} \alpha$ appear as dark/ absorbing structures, while they are bright structures in emission in C IV. According to the low spatial resolution of the instruments, both structures occupied the same area and at the base of the surge, a bright point was observed in CIV and $\mathrm{H} \alpha$ (Schmieder et al., 1982; Schmieder et al., 1983). In these former studies of a surge occurring on 2 October 1980, its lifetime was around 20 min with upflows followed by downflows, with a radial velocity reaching $60 \mathrm{~km} / \mathrm{s}$ in both lines $\mathrm{H} \alpha$ and $\mathrm{C} \mathrm{IV.}$ Upflows and downflows were registered successively and not simultaneously such as in rotating jets. However, the wide line profiles in C IV and $\mathrm{H} \alpha$ could indicate that along the line of sight, opposite flows exist in unresolved structures. Nevertheless, the large widths of C IV line profiles were explained by high microturbulence of $120 \mathrm{~km} \mathrm{~s}^{-1}$. In this context, the microturbulence is the nonthermal microscopic component of gas velocity in the formation zone of spectral lines. It is frequently used to explain broadened line profiles in the stellar spectra. $\mathrm{H} \alpha$ Dopplershifts (radial velocity) were computed with the cloud model technique ( $\mathrm{Gu}$ et al., 1996; Mein et al., 1996) and the horizontal speed by following the leading edge of surges. Measuring Dopplershifts supports the idea that in surges, there are mass motions and not propagating waves. During the initiation phase of surges, flow acceleration was established in $\mathrm{H} \alpha$ and $\mathrm{C} \mathrm{IV}$, which permitted the authors to conclude that pressure gradients could be the driving force of the surge. However, the acceleration phases were different in both lines which means that C IV emission did not come from the transition region of surges but from independent structures. C IV surge quantities (velocity and acceleration) varied on a very short time scale as if there were pinched zones in the magnetic tube. In $\mathrm{H} \alpha$, the displacement of the maximum velocity was observed along the axis of the surge. In C IV, the velocity maxima are observed at given distances along the surge strong upward velocity maximum followed by low velocity with no propagation during the evolution of the surge, suggesting the existence of kink waves. This kind of behavior for surges observed in transition region temperature has not been repeated but has been observed and modeled for spicules (He et al., 2009). A Dopplershift signature with blue and red shift from one edge to the radially opposite edge for a given surge cross-section was interpreted as torsional waves. However in these earlier observations, the authors favored the interpretation of successive upflows and downflows, implying no rotation. As noted in the chapter, 3.1 torsional waves seem to be more frequently observed. Torsional waves are now used in MHD simulations as drivers of jets (Pariat et al., 2015).

Radio Type III bursts were also often associated with surges, suggesting that surges followed open magnetic field lines or very large loops (Chiuderi-Drago et al., 1986; Kundu et al., 1995). This idea has been confirmed by using NLFFF extrapolation showing how nonthermal types III associated with jets escape along open field lines at the edge of close structures over active regions ( $\mathrm{Lu}$ et al., 2019; Mulay et al., 2019). Schmieder et al. (1983), Schmieder et al. (1984) reported on the recurrence of $\mathrm{H} \alpha$ and C IV surges with a time delay between two jet ejections of 15-30 min. They proposed that such recurrent ejections could be due to periodic energy storage and periodic reorganization of magnetic field as envisaged to occur for flares, but at lower energy levels.

\subsection{Energy Budget in Surges and X-Ray Loops}

The energy budget was determined by analyzing the signatures of surges and jets in multiwavelengths and multitemperatures obtained by the instruments on board the Solar Maximum Mission (SMM) launched in 1980. Coordinated campaigns with ground-based instruments allowed simultaneous observations in $\mathrm{H} \alpha$ with the MSDP operating on the solar tower in Meudon, in $\mathrm{O} \mathrm{V}$ and Fe XXI with the UVSP/SMM, and in soft X rays with the HXIS/SMM (Schmieder et al., 1988; Schmieder et al., 1993; Schmieder et al., 1996b). The cool (H $\alpha)$ and warm $(\mathrm{O} \mathrm{V})$ surge plasma show velocities of the order of $120 \mathrm{~km} / \mathrm{s}$ in a comb-shaped surge observed on 11 November 1980 at the edge of a sunspot (Schmieder et al., 1988). The surge intensity ( $\mathrm{H} \alpha$ and $\mathrm{OV}$ ) was well-correlated with the emission of an associated loop observed in the HXIS channel (3.5-5.5 keV) and in the FeXX1 line (UVSP), one footpoint of the loop being close to the footpoint of the jet (Figure 1). This suggests that the surge could be due to the reconnection between the closed loop and open field. The association of X-ray and UV emission with $\mathrm{H} \alpha$ surges allowed the authors to estimate the energy budget between kinetic, potential, and radiative energy. The potential and kinetic energy was both of the order of $2.5-510^{28}$ ergs, two orders larger than the radiative loss in the X-ray loop. They concluded that the magnetic energy liberated at the base of the surge was mainly transferred to kinetic energy, and only a small part was released in thermal and nonthermal energy. In a 


\section{A}

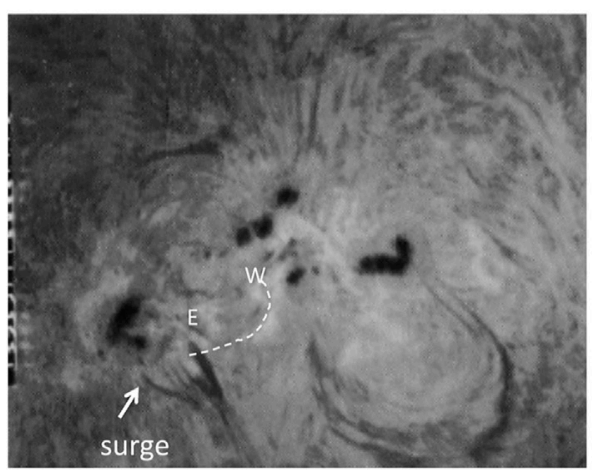

B

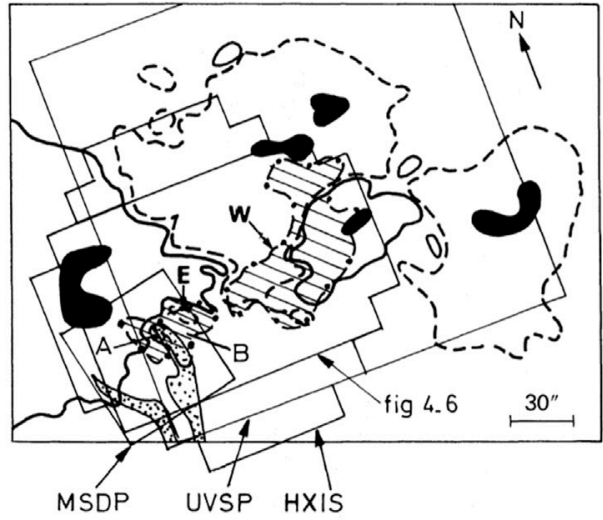

FIGURE 1 | Surge in $\mathrm{H} \alpha$ observed on 11 November 1980, with the Solar Optical Observations Network (A) and all the signatures of the event obtained by the instruments (UVSP, HXIS) on board the SMM (B). The surge in $\mathrm{H} \alpha$ from the MSDP operating in the Meudon solar tower and in O V from the UVSP is indicated by the dotted area, the bright $\mathrm{H} \alpha$ areas at its footpoint by the letters $\mathrm{A}$ and $\mathrm{B}$, the hatched area represents the footpoints (E, W) of a loop observed in soft $\mathrm{X}$ ray (3.5-5.5 eV) with HXIS and Fe XXI with the UVSP. The EW loop is drawn with a dashed line in the H $\alpha$ image [adapted from Schmieder et al. (1988)]

successive study, analyzing different multiwavelength data (Schmieder et al., 1996b), the authors concluded that the magnetic reconnection should occur in the corona. The energy is transported by energetic particles along the loops. The energetic particles are losing energy in the chromosphere as surges in open field lines and as bright loops in close loops such as for miniflares. The X-ray spikes appear earlier than $\mathrm{H} \alpha$ and UV surges. The maximum upward velocity happens 10 minutes after the onset of the surge. The response of the chromosphere depends on magnetic topology.

The partition of magnetic energy released as kinetic or thermal energy during reconnection between close loops and open structures is still not clear. A statistical analysis of the relationship between miniflare and jets shows no positive answer and exhibits broad distributions of the delay between these two events and of their amplitudes (Musset et al., 2020). This confirms the result of this historical study that flares and associated jets belong to a global system where the energy is released during reconnection and the partition depends on the magnetic configuration of the system (close and/or open structures). In the study by Musset et al. (2020), the delay between the nonthermal X-ray peak emission and the peak intensity of the jet is negligible, which implies that the jet could be produced by magnetic reconnection. However, this does not give any information on the magnetic configuration. Pressure pulses can also be created by magnetic reconnection which may release impulsively energy and heats the plasma in closed and open flux tubes. In the standard model of magnetic reconnection for eruption, the chromosphere plasma is heated and evaporates.

In closed flux tubes, the density increases, and strong pressure and temperature gradients produce upward motions (Shimojo et al., 2001). Indeed pressure-driven upflows are slow; they correspond to trans-sonic flows. This model may still be valid for surges but is not applicable to fast X-ray jets. This concept of pressure gradient for initiating surges has been discussed in the context of the evaporation model and criticized because it requires heating the chromosphere to transition zone temperatures and then cooling. However, the cooling time is very short at these temperatures with no delay (Schmieder et al., 1994).

\subsection{X-Ray Jets}

The Yohkoh satellite launched on 30 August 1991 with on board the SXT instrument (Tsuneta et al., 1991) helps us to make definitively progress on X-ray jets and to decide if jets was initiated by pressure pulse (Sterling et al., 1994) or by magnetic reconnection (Shibata et al., 1992; Shibata et al., 1994; Shibata et al., 1996). The former authors defined X-ray jets as transitory X-ray enhancements with collimated motions. All the jets are associated to microflares. Their length is 1,000 to 4 $\mathrm{x} 10^{5} \mathrm{~km}$. Their apparent speed is $10-1,000 \mathrm{~km} / \mathrm{s}$ and the temperature 4-6 MK. The morphology of X-ray jets shows converging shape (lambda-shaped), suggesting a null point near the footpoint of the jet. Parasitic polarities are often observed in the footpoint favoring magnetic reconnection, and this fact gives evidence of a null point. Surges could accompany X-ray jets (Schmieder et al., 1996a; Canfield et al., 1996). In the former study, the association with the jet was an X-ray loop and not a fine X-ray jet similar to the previous observations with HXIS/SMM (Schmieder et al., 1996b). The hot footpoint is not always exactly at one end of the jet and could be represented by a loop.

A unified reconnection model valid for flares and jets called plasmoid-induced reconnection was proposed by Shibata (Yokoyama and Shibata, 1995; Shibata, 1999) (Figure 2A, panels $\mathrm{c}$ and $\mathrm{d}$ ). The standard CSHKP model and the emerging flux model were compatible with this plasmoidinduced reconnection, where plasmoids were compared to the flux rope ejected during flare. Therefore, they proposed that reconnection occurred between the plasmoids and the ambient field, and hot loops formed below similar to post-flare loops as in 
A

(a)

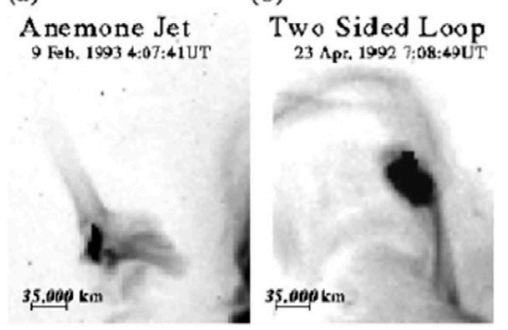

(c)

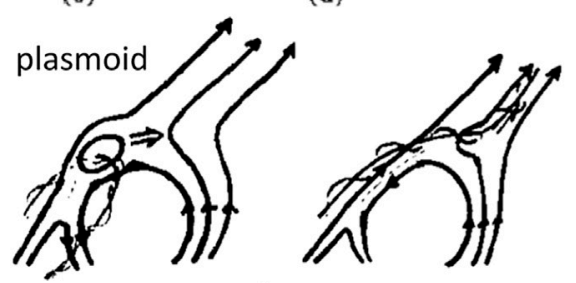

B
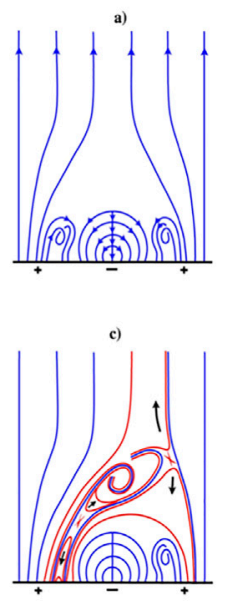

Twisting arcades SDO +IRIS
C
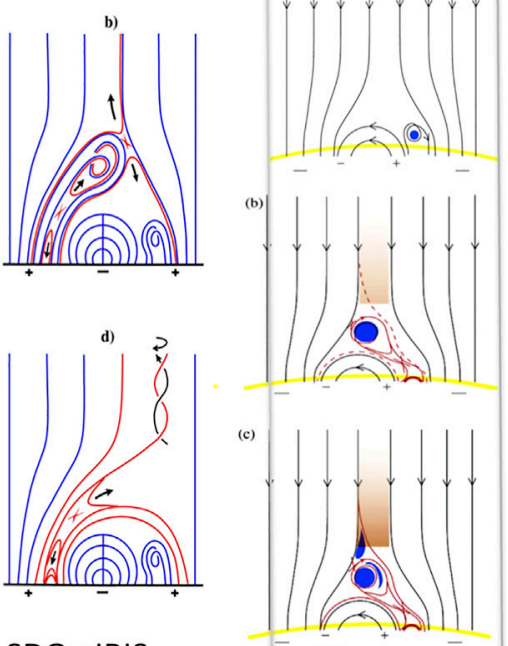

Filament

FIGURE 2 | Cartoons representing the initiation of jets. Left column (A): (panel a) two kinds of X-ray jets observed with Yohkoh: anemone jet and two-side loop jet, panel (b) unified CSHSP model of flares and flux emerging model: the plasmoid-induced reconnection model proposed by Yokoyama and Shibata (1995); Shibata (1998) [adapted from Shibata (1999)]. The plasmoid is a magnetic island or a twisted flux rope. Middle column (B): a possible formation mechanism of penumbral jets from the eruption of a magnetic arcade (the core of which becomes a twisted flux rope as it erupts) inserting shear and twist in the jet spine. In this sketch, only the "cross-cut/ section" across the central axis of the tail of the penumbral filament is represented so that the field in the filament head is behind the viewer (in the line of sight) [adapted from Tiwari et al. (2018)]. Right column (C): blowout jet initiated by a mini filament; in panel (b), two red crosses indicate 2 sites of reconnection: the low cross corresponds to tether cutting reconnection and a bright point loop forms below; the top cross corresponds to break-out [adapted from Sterling et al. (2015); Sterling and Moore (2020)].

the standard flare model. Simulations in 2 and 2.5 dimensions develop possible models based on the conceptual idea that magnetic reconnection may accelerate plasma in two ways (Shibata and Uchida, 1985; Shibata and Uchida, 1986; Shibata et al., 1996). With the tension-driven model, plasma is accelerated to Alfvénic velocities in the vicinity of the reconnection site as a sling-shot mechanism (Yokoyama and Shibata, 1996; Moreno-Insertis et al., 2008). The second way is characteristic of the initial magnetic configuration: the untwisting model in which the closed magnetic structures should possess initially shear or twist (Schmieder et al., 1995; Canfield et al., 1996; Jibben and Canfield, 2004). These two concepts are important in the acceleration process of jets to reach Alfvénic velocities or sub-Alfvénic velocities. Recent studies appear to favor the shear inside the embedded structure from which jets will be initiated (Kumar et al., 2019). In 3D simulation, it is clear that plasmoids are created during reconnection and are ejected along current sheets as it is shown in the study by Kumar et al. (2019). In $3 \mathrm{D}$, the curvature of magnetic field lines can be in both directions; therefore, the distinction of these two mechanisms is not clear. In $3 \mathrm{D}$, both mechanisms contribute simultaneously to driving plasma from reconnection sites. Some amount of shear or twist in one component of the reconnecting flux systems is needed to provide enough free energy for the eruption/jet; otherwise, as many models have shown, only weak jets result.

\subsection{New Instruments (2000-2010)}

Later, high spatial resolution instruments were developed and brought new imaging and spectral observations to fore. Solar jets were observed in different regions of the Sun: network, coronal hole, active region, in the chromosphere, and in the corona. Theory and interpretation were rapidly developing. We just list the new generation instruments with some relevant studies, such as the Swedish solar telescope (SST) (Nóbrega-Siverio et al., 2017), the NST/GST at the Big Bear Observatory (Kumar et al., 2015), the TRACE mission with its UV instrument (Alexander and Fletcher, 1999), Hinode with its SOT polarimeter, and its spectrograph EIS (Muglach, 2021) and XRT (Madjarska, 2011) showing multiwavelength jets with different spatial, physical, and temporal properties in coronal holes and quiet Sun. Hinode Review Team (2019) (section 7) summarize significant progress with insightful observations using the advanced instruments (e.g., EIS, SOT, and XRT) (Cirtain et al., 2007; Savcheva et al., 2007; He et al., 2009).

For example, we may note the detection of the excitation and launch of kink waves due to magnetic reconnection. Another example of the merit contributed by EIS and XRT for the coronal jet study was the observation of a mini-CME (He et al., 2010). Time-varying Dopplergrams of a mini-CME event were successfully captured and recorded by EIS when it was repetitively rastering the same solar region with a repetition period of about $6 \mathrm{~min}$. The initial eruption speed was estimated to be as low as $30 \mathrm{~km} / \mathrm{s}$ from the Dopplergram. The 


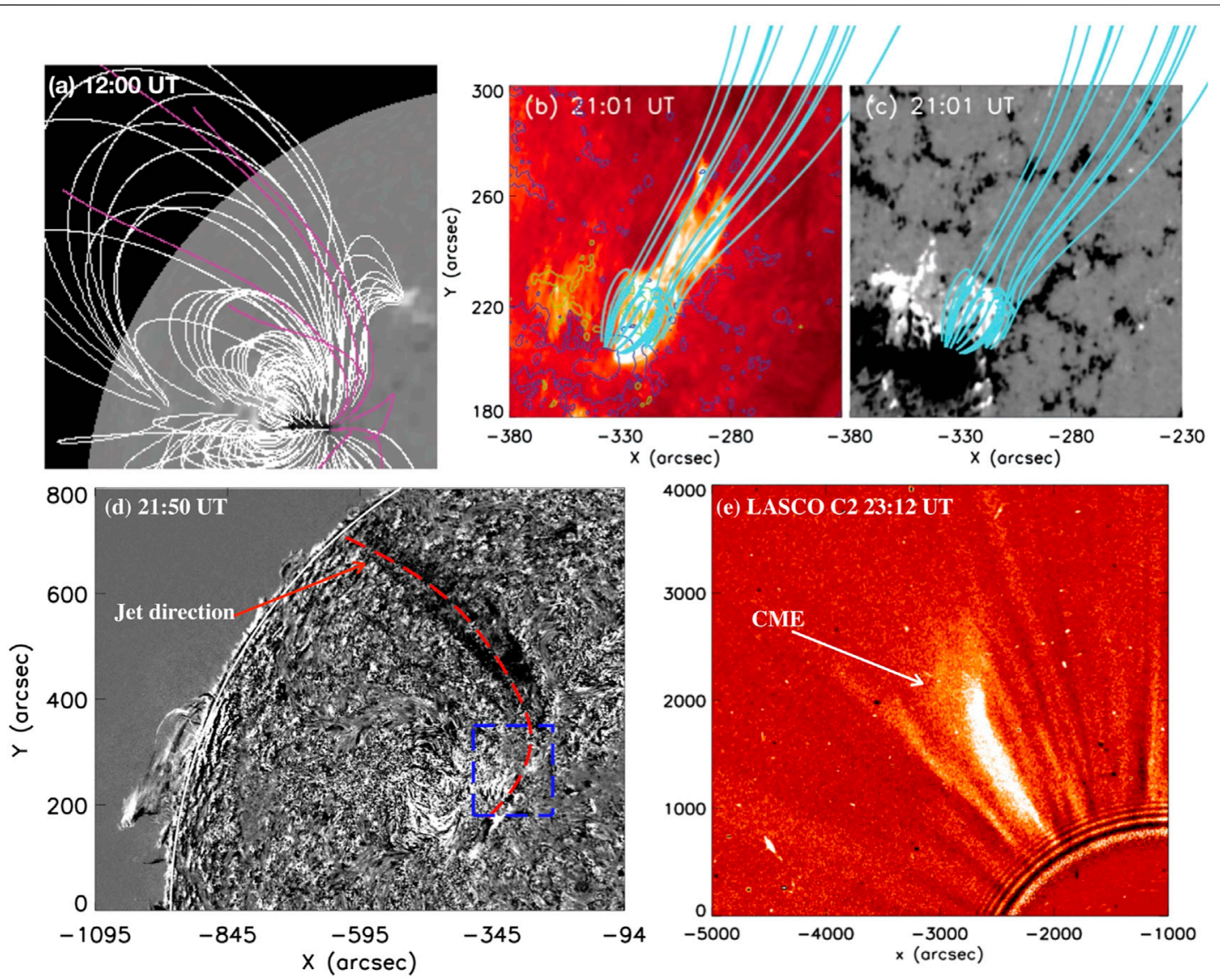

FIGURE 3 | Jet initiating a narrow CME: PFSS extrapolation of the large field of view (FOV) is shown in panel (A). The white and pink lines are the closed and open magnetic field lines at the jet location. The open field lines mirror the jet propagation from its source to the solar corona, which is indicated by a curve (dashed red line in panel (D). In panels (B) and (C) are presented the AIA $304 \AA$ jet observation and the HMI magnetogram, respectively, showing the jet source. The cyan lines are the magnetic field lines from the source region, which shows a closed structure at the jet base and open lines afterward. Panel (E) shows the narrow CME observed by LASCO C2 (adapted from Joshi et al. (2020c).

associated X-ray emission of the mini-CME started from a sudden brightening at one footpoint of the closed loop and then a rapid propagation of the brightening along the erupting closed loop. This loop could represent the onset of a mini-CME.

Later on, many examples showed that collimated jets can produce coronal mass ejections observed with coronagraphs, for example, SOHO/LASCO (Figure 3) (Panesar et al., 2016a; Sterling et al., 2018; Joshi et al., 2020c; Kumar et al., 2021) and acceleration of particles (Mulay, 2018; Joshi et al., 2021a).

In the following years, the more important achievement was due to the development of theory to reply to the following questions: what is the driver of jet? What is the relationship between jet and surge? What are the physical conditions of jet and surge? We focus the next sections on recent observations using the SDO and IRIS. This development has its seeds in the observations of many jets after the launch of the SDO in 2010 and more recently the launch of the Interface Region Imaging Spectrograph [IRIS -De Pontieu et al. (2014)] in 2013.

In Section 2 are presented the results of SDO observations which inspire the development of 3D MHD simulations based on flux emergence as the trigger of jet. In Section 3 are presented twisted jets observed by the SDO and IRIS and their interpretation using 3D simulations based on the existence of the transfer of twist.

\section{SDO AND THE ONSET OF JETS}

In 2010, the SDO was launched with two important instruments onboard: the Atmospheric Imaging Assembly (AIA: Lemen et al., 2012), providing extreme ultraviolet (EUV) and ultraviolet (UV) data, and the Helioseismic and Magnetic Imager (HMI: Scherrer et al., 2012), providing magnetograms both with a high spatial resolution ( 0.6 and $0.5 \mathrm{arcsec}$ ) and high temporal cadence (12 and $45 \mathrm{~s}$ ). A very impressive number of publications concerning jets observed with AIA with more precise results of their characteristics and their triggers appeared (Raouafi et al., 2016; Shen, 2021). In the review of Hinode Review Team (2019), there is not only an interesting discussion of the jets observed with the Hinode instruments but also a deep discussion on the origin of the jets observed with the SDO/AIA. 


\subsection{Morphology of Jets}

With Yohkoh observations, coronal jets were classified into two types: straight anemone jets and two-sided loop jets (Figure 2A, panels a, and b) (Shibata et al., 1994). Anemone jets consist of a collimated jet and a dome-like base corresponding to magnetic flux emergence. The two-sided loop jets exhibit diverging flows from their excitation center. This new kind of jet was also observed with Hinode/XRT and EIS instruments, which confirmed that the opposite direction flows via Dopplershifts (Sterling et al., 2019).

With the SDO/AIA, a new kind of jet was discovered called blowout jets (Moore et al., 2010). Compared to standard jets, they exhibit different characteristics: an additional bright point inside the dome, a blowout eruption of the base arch that could host a twisting mini-filament, and an extra jet-spire strand close to the external bright point. The probability of a jet to be a blowout jet was found to reach 50\% (Moore et al., 2013; Chandra et al., 2017). They look like break-out eruptions initiating CMEs (Joshi et al., 2020a; Kumar et al., 2021).

Several observations show that mini-filament eruptions are closely associated with coronal jets and could be the triggers of blowout jets as in large-scale filament eruptions before flares (Figure 2C) (Shen et al., 2012, Sterling et al., 2015; Shen et al., 2017). New cartoons have been proposed for the magnetic configuration for jets with a filament close to an emerging flux (Figure 2C) (Sterling et al., 2018; Sterling and Moore, 2020) and for jets in penumbra with multi-arcade configuration (Figure 2B) (Tiwari et al., 2018). In these observations, the origin of the jets is identified in magnetic flux cancellation rather than in flux emergence (Moore et al., 2010; Panesar et al., 2020). Kumar et al. (2019) analyzed 27 jets in equatorial coronal holes using SDO/AIA observations and found a high proportion of jets involving filament channel eruption and free energy resulting from shear motions or pre-existing twist, with no evidence of flux cancellation.

\subsection{Flux Cancellation}

Several studies show clearly that tiny coronal jets in coronal holes and quiet Sun are due to flux cancellation (Savcheva et al., 2007; Panesar et al., 2016b, Panesar et al., 2017, Panesar et al., 2018a; McGlasson et al., 2019). In coronal holes, converging flows toward the boundary of super-granule with mixed polarities leading to cancelling flux was found to be a favorable solution to explain the onset of jets (Young and Muglach, 2014; Muglach, 2021). McGlasson et al. (2019) made some statistics on 60 such coronal jets observed with the AIA 171 filter and found that nearly all are associated with dark absorbing features between two opposite polarities considered as proxies of mini-filaments. Jets were formed by flux cancellation. They said that it is the result of lower reconnected loop submergence into the photosphere. Two bright points were observed: one internal brightening and one external brightening with extended magnetic field lines along which the jet was running. By analogy with the cartoon in Figure 2C, they discussed that these two brightenings may correspond to the two reconnection points of this scheme. These coronal jets lasted around $10-12 \mathrm{~min}$. Their bases are small between $8,000 \mathrm{~km}$ and $17,000 \mathrm{~km}$ in the case of the 10 coronal jets analyzed by Panesar et al. (2016b). Similar pattern of jets at the edge of the network were also observed simultaneously in AIA $171 \AA$ and in the slit-jaw images of the IRIS 1400 filter containing Si IV lines (Panesar et al., 2018b). They were triggered by the reconnection due to cancellation.

However, the identification of mini-filaments is sometimes questionable because an arch filament system (AFS) over emerging flux looks like a mini-filament (cool material). However, their formation and magnetic configuration are completely different. A mini-filament is found along the inversion line (PIL) between positive and negative polarities, and the AFS is perpendicular to the PIL and unsheared; they are not twisting filaments, so there is no free energy in that case. The mini-filament should be detected along the PIL between the dome of the emerging flux and the ambient field as shown in the cartoon in Figure 2C. We show in subsection 2.3 an example of possible misinterpretation.

The detection of cancellation of the flux depends crucially on the spatial resolution of the magnetograms, and certainly the HMI is not sensitive enough to detect small dispersed magnetic field and validates real cancellation of flux. Reconnection between magnetic field lines is due to the motions of their footpoints induced by convection. Therefore, reconnection may occur in the whole corona depending on the magnetic topology of the region. Kumar et al. (2018) showed jet onset resulting from explosive breakout reconnection between the flux rope inside the closed structure and the external open field in a classic fan-spine magnetic topology, characterized by a slowly rising EUVbright sigmoid and mini-filament, dimmings at both ends of the sigmoid, weak quasi-periodic outflows at the null, and multiple plasmoid formation in the flare current sheet beneath a rapidly rising flux rope. There was no evidence of flux emergence or cancellation up to $16 \mathrm{~h}$ before the impulsive event. For this case, the observed features closely matched the predictions of breakout-jet models (Wyper et al., 2018; Wyper et al., 2019).

\subsection{Flux Emergence, Null Point, and Bald Patch}

Concerning magnetic flux emergence, it is currently accepted that a null point or separator is formed between the emergence and the surrounding magnetic field. During reconnection at the null point, energy can be released in the form of a flare, eruption, or jet (Filippov, 1999). In the corona, the magnetic field is free and frozen into the plasma almost everywhere. Only at null points and in current sheets (frequently present in separatrices) can the energy release occur. A magnetic field configuration with separatrices can favor occurrence of jets. For example, it was shown that magnetic field lines over the emerging flux of a bipole close to a sunspot could reconnect to the ambient open magnetic field lines via bald patch (BP) regions (Guo et al., 2013; Chandra et al., 2017). BPs are regions where the magnetic field lines are tangential to the solar surface (see Figures 4A,C, where blue/red lines are for the prereconnected/reconnected magnetic field lines, respectively). Blue magnetic field lines bend toward the photosphere as they are attracted by opposite polarities. They 

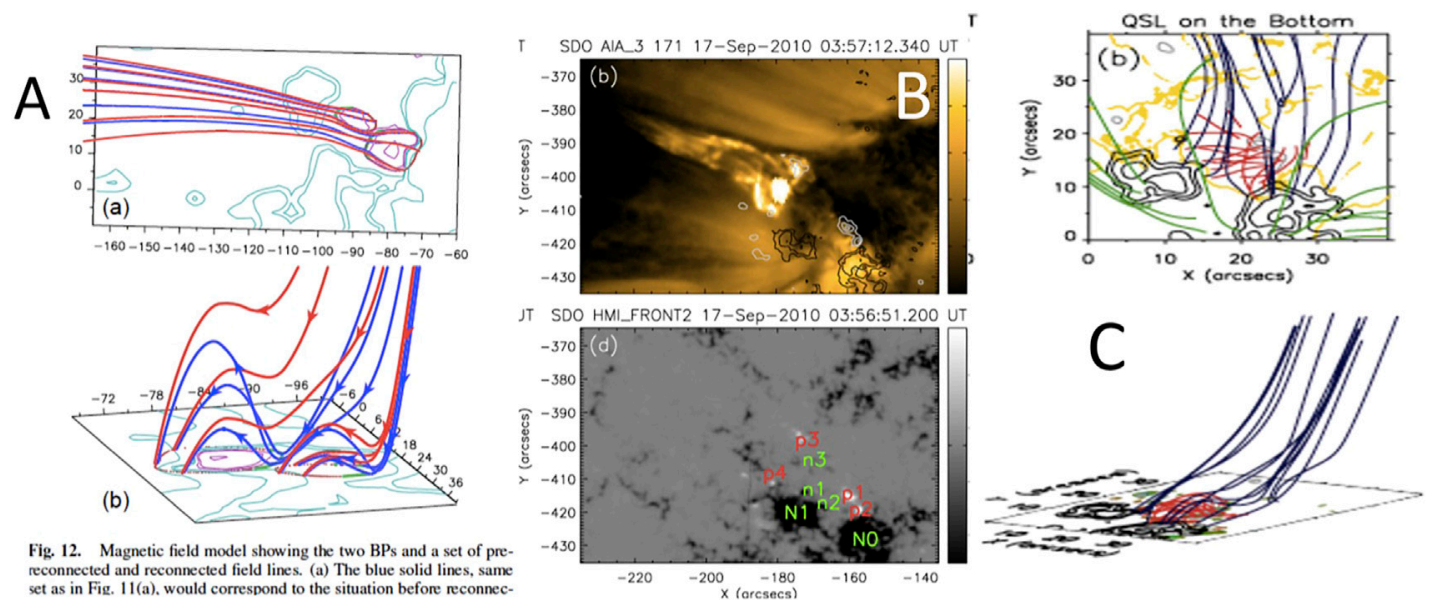

reconnected and reconnected field lines. (a) The blue solid lines, same
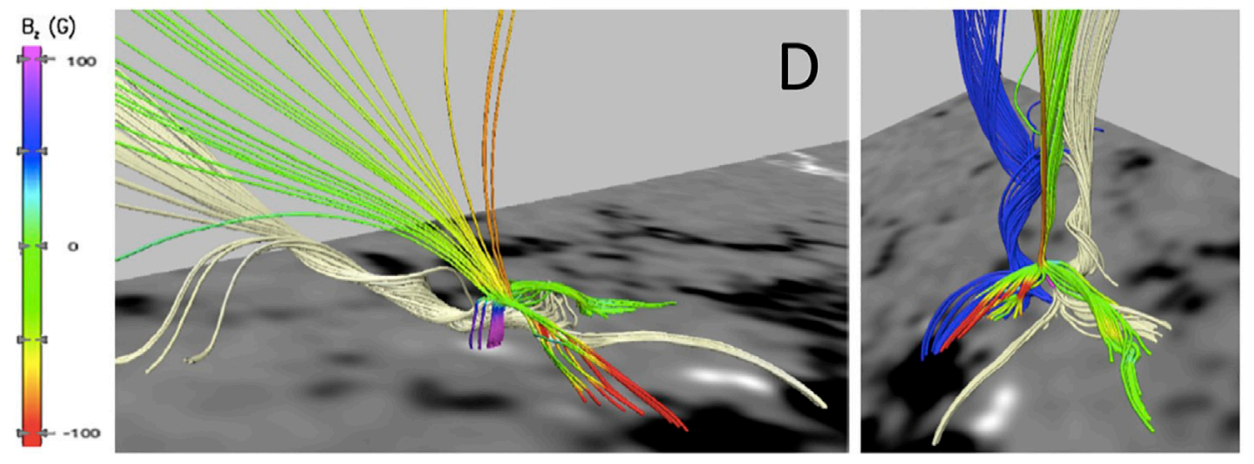

FIGURE 4 | Magnetic reconnection initiating solar jets in bald patch (BP) and in null point. (A) Reconnection by bald patches, viewed from above and side view [from Chandra et al. (2017)]; (B) Jet observed by the AIA $171 \AA$ filter and the corresponding HMI magnetic map; (C) NLFF extrapolated magnetic field lines with bald patch region and electric currents [from Guo et al. (2013)]. (D) NLFF extrapolated magnetic field showing bald patch and null point [from Schmieder et al. (2013)].

are pinched together and reconnect to form the red magnetic field lines which are no more tangential to the solar surface. In a $\mathrm{BP}$, the magnetic field appears to be going from negative to positive polarity contrary to loops where magnetic field is going from positive to negative. With shear motions, the two branches of the BP insert a thin layer like in separatrix, and electric currents are stored until reconnection occurs and releases the energy.

In the study by Guo et al. (2013), a jet observed on 17 September 2010 by the AIA and HMI expanded in $10 \mathrm{~min}-100 \mathrm{Mm}$ in length with a speed of $200 \mathrm{~km} / \mathrm{s}$ and a large base. BPs have been found in a nonlinear force-free extrapolation of photospheric magnetograms. It was proposed that magnetic reconnection could occur at the BP separatrices. During the reconnection, cool plasma could be ejected along open field lines driving jets. This kind of evolving magnetic structure called separatrices or quasi-separatrix layers (QSL) are known to be the location of drastic changes of connectivity, and narrow current layers are created along them (Démoulin et al., 1996). In the case of Guo et al. (2013) QSL footprints with electric current were detected around the emerging bipole base of the jet close to the main polarity (see Figure 4C). The recurrence of the jets was co-temporally related to the accumulation of electric currents. In this study, it was shown that these jets could be explained by flux emergence (Shibata, 1998) and also by the converging flux model (Priest et al., 1994) since the newly emerged magnetic flux is consistent with the former model and the bald patch configuration is consistent with the latter one. But both models are two dimensional with magnetic reconnection in separatrices (as implied by their dimensionality), while the magnetic connectivity is not necessarily discontinuous in the three-dimensional space. The abovementioned models can be generalized as a three-dimensional configuration with a magnetic null point (e.g., Moreno-Insertis et al. (2008); Török et al. (2009); Pariat et al. (2009); Wyper et al. (2018).

Revisiting these data and analyzing carefully the nonlinear force-free extrapolation, BP separatrices, low-altitude flux ropes, and even a null point were identified at the base of the jet (see Figure 4D) (Schmieder et al., 2013). Therefore, we conclude that it is difficult to identify clearly in 3D magnetic field extrapolations the exact regions where energy is evacuated. Commonly, many low-altitude null points and separatrices exist and are the possible sites of triggering jets and eruptions. Nevertheless, while standard or Eiffel tower-shaped jets appear to be caused by reconnection in current sheets containing null points, reconnection in regions containing bald patches, such as the jet of Guo et al. (2013); Joshi et al. (2020b), seems to be of prior importance for triggering the jet. 

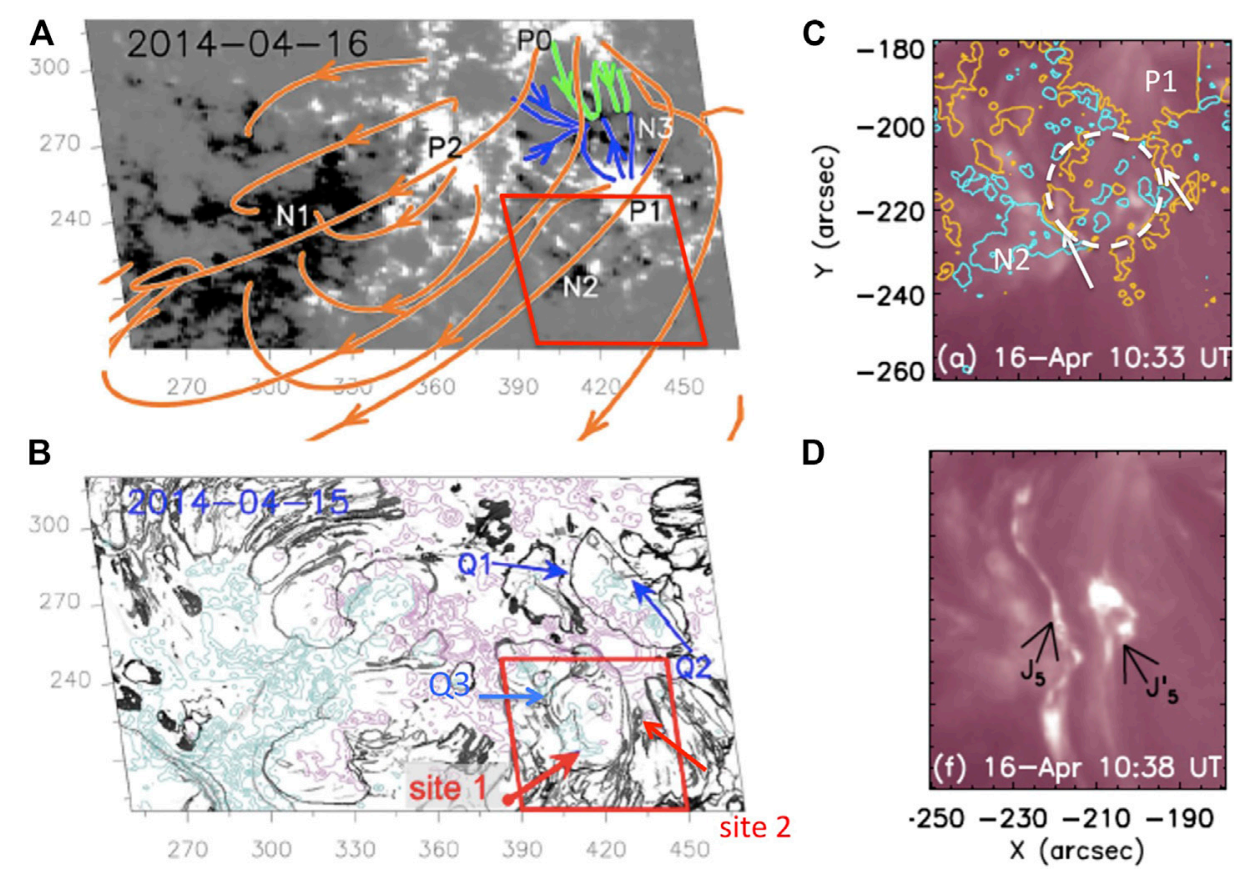

FIGURE 5 | Active region NOAA 12035 observed on April, 15-16 2014, (A) HMI magnetogram overlaid by extrapolated magnetic field lines, (B) quasi separatrix layers (QSL) over the magnetic field contours; the red box indicates the two sites of the reconnection of the jets (red arrows). (C) Zoom on the magnetic region formed by mixed emerging polarities (inside the white circle) between polarities P1 and N2. (D) Two parallel jets with origin in the two sites [adapted from Joshi et al. (2017)].

The question is, what is the role of flux emergence compared to flux cancellation role? In view of this divergence of opinions about the main trigger of the jets, it is difficult to determine which mechanism dominates (Schmieder et al., 2014). In fact, the main trigger is the convection which moves the magnetic field lines in the solar atmosphere and leads to reconnection. Convection is responsible for emergence, cancellation, shear, and twist and finally generates free energy. Motions of footpoints of magnetic field lines allow accumulating electric current in the special loci where magnetic field can change easily of connectivity in the QSLs. Before jet onset, bright points are frequently observed in the footprints of the QSLs. When enough energy is stored as was shown with the measurement of electric currents (Guo et al., 2013), the release of energy produces kinetic phenomena, such as jets or eruptions. In the breakout model, reconnection between the flux rope below the breakout current sheet can produce very energetic events.

\subsection{Case Study of the Emerging Flux on the Disk}

This section explains how, using the same data of jets observed with AIA and HMI, two different groups concluded differently on the trigger of the jets. One group explains the jets as slipping reconnection around flux emergence (Joshi et al., 2017). The second group explains the jet as a blowout jet driven by the eruption of a mini-filament (Shen et al., 2017). In fact, 11 recurring solar jets originated from two different sites (site 1 and site 2 ) close to each other (about $11 \mathrm{Mm}$ ) in the NOAA active region (AR) 12 ,035 during 15-16 April 2014 (Joshi et al., 2017). The analysis of the active-region magnetic configuration showed that a strong bipole (P2-N2) emerged on April 152014 inside a remnant active region (P1-N1) (Figure 5A). In the neighborhood of P1, flux emergence continuously occurred between P1 and N2, and a circle-shaped quasi separatrix layer (QSL) was detected around these new emerging polarities (Figures 5B,C). On 16 April, both sites were located in QSLs, Flux emergence and cancellation mechanisms triggered the 11 jets in site 1 and/or site 2 . The jets of both sites had parallel trajectories and moved to the south with a speed between 100 and $360 \mathrm{~km} \mathrm{~s}^{-1}$. The jets of site 2 occurring during the second day had a tendency to move toward the jets of site 1 and merge with them. It was conjectured that the slippage of the jets could be explained by the complex topology of the region, which included a few low-altitude null points and many quasi-separatrix layers (QSLs), which could intersect with one another.

Only one of these jets at 07:40 UT has been analyzed by Shen et al. (2017) using the SDO and the New Vacuum Solar Telescope (NVST - Liu et al. (2014)) in Fuxian lake in China. Their interpretation of the trigger of this jet is different from the series of jets described above. Effectively with the high resolution of the NVST, they could detect in $\mathrm{H} \alpha$, a dark absorbing feature perpendicular to the jet direction. They explained the event as a blowout jet, like a mini eruption driven by the fibril that they call mini-filament. The jet is mainly observed in hot plasma (emission in the AIA 171), with no cool jet visible in absorption as the other series. It would mean that the active and complex emerging flux close 


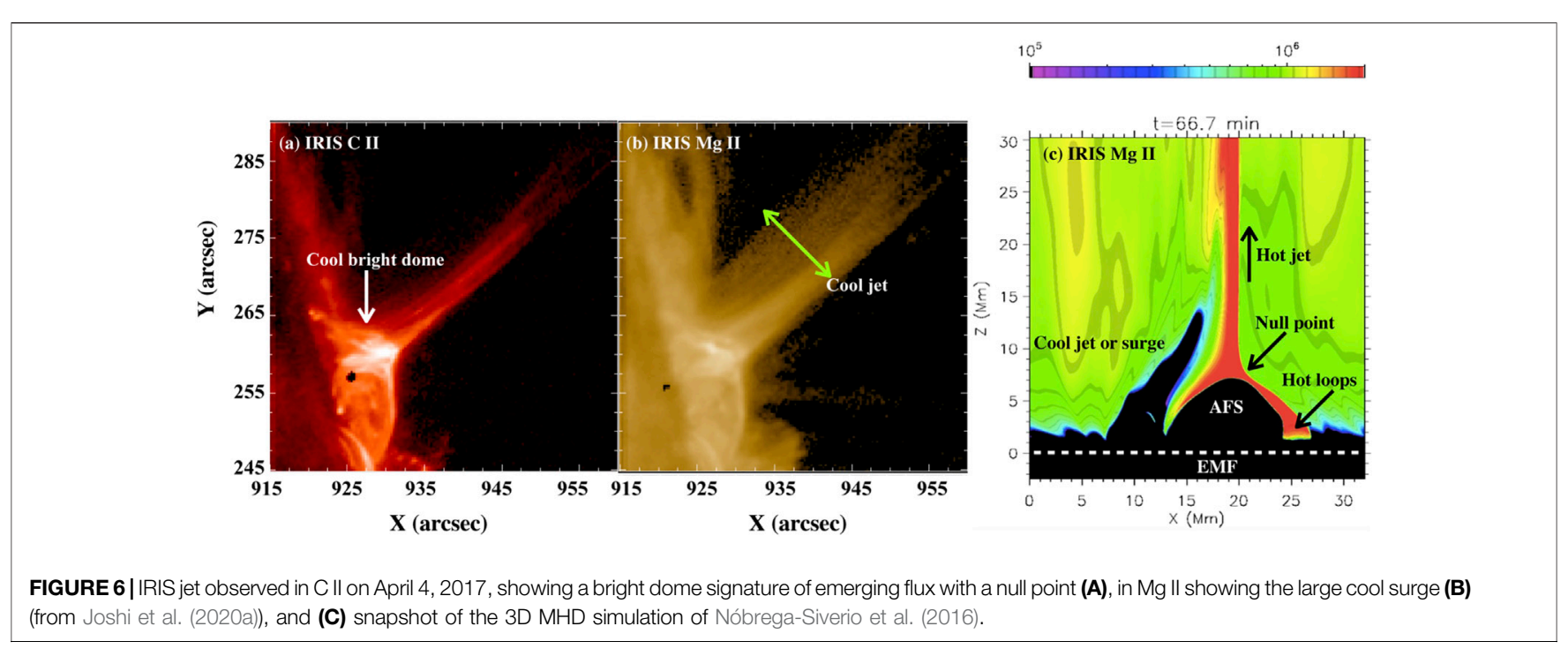

to the sunspot triggers the recurrent jets in different ways. The site of reconnection is already slipping along the QSL, so null point reconnection could be more important for this blowout jet that is concerned. On the other hand, the dark structure could also be an arch filament system and not a real mini-filament with no twist. The interpretation of observations is very complex, and we need more and more high-resolution instruments.

\subsection{Case Study of the Emerging Flux at the Limb Viewed in 3D MHD Simulation}

Six recurrent jets occurring in the active region NOAA12644 on April 4, 2017 were observed in all the hot filters of AIA as well as cool surges in IRIS slit-jaw high spatial and temporal resolution images (Joshi et al., 2020a). The hot jets are collimated ejections observed in the hot temperature AIA filters (Figure 6); they have high velocities (around $250 \mathrm{~km} / \mathrm{s}$ ) and are accompanied by cool surges and ejected kernels that both move at about $45 \mathrm{~km} / \mathrm{s}$. This series of jets and surges provide a good case study for testing the 2D and 3D magnetohydrodynamic (MHD) emerging flux models (see the numerical simulations of Török et al. (2009); Archontis et al. (2004); Moreno-Insertis et al. (2008); Moreno-Insertis and Galsgaard (2013). In their simulations, they solved the MHD equations in three dimensions to study the launching of coronal jets following the emergence of magnetic flux.

The jet observations at the limb infer clearly a null point in the corona and a dome below in all the AIA filters. The doublechambered structure of the dome corresponds to the regions with cold and hot loops that are in the models below the current sheet that contains the reconnection site (Nóbrega-Siverio et al., 2016). The former model is based on the radiation-MHD Bifrost code (Gudiksen et al., 2011). In the 3D models, the jet is launched along open coronal field lines that result from the reconnection of the emerged field with the pre-existing ambient coronal field. Underneath the jet, two vault structures are formed: one containing the emerging cool plasma and the other a set of hot, closed coronal loops resulting from the reconnection. The cool surge with kernels is comparable with the cool ejection and plasmoids that naturally appear in the current sheet in models $(\mathrm{Ni}$ et al., 2021).

The comparison of the observations of the structures and time evolution of the jet complex observed at the limb with numerical experiments of the launching of jets following flux emergence from below the photosphere shows significant similarities, proving that such a $3 \mathrm{D}$ experiment is valid to explain the AIA observations. Quantitatively, the characteristics of the jets (speed and temperature) fit well with the values determined in the MHD simulations (Nóbrega-Siverio et al., 2016). The comparison of this case study with a model of emergence strongly suggests that this jet may have been initiated by flux emergence.

Another example of flux emergence was studied by Yang et al. (2018), showing a comprehensive force analysis of the cool and warm jets. The cool jet was mainly accelerated by the gradients of both thermal and magnetic pressures near the outer border of the mass-concentrated region, which is compressed by the emerging loop, while the hot jet was accelerated mainly by the sling-shot effect (curvature tension of magnetic field) of reconnected magnetic field lines and heated directly by resistive dissipation.

\section{UNTWISTED JETS AND MODELS}

\subsection{Rotating Structure}

Helical or rotating jets are frequently observed in AIA $304 \AA$ and in X-ray (Nistico et al., 2009) and in the multichannels of AIA (Kumar et al., 2018). Rotation rate and speed have been estimated by following some fine structures in the jet (Chen et al., 2012; Hong et al., 2013). Helical jets have been reconstructed in 3D by using STEREO spacecraft (Patsourakos et al., 2008). In the study of Schmieder et al. (2013), time-slice analysis along a jet revealed a striped pattern of dark and bright strands propagating along its axis, with apparent damped oscillations across the jet (Figure 4 panel b). This was suggestive of a (un)twisting motion in the jet, possibly an Alfvén wave. Later, similar twisting was shown by 


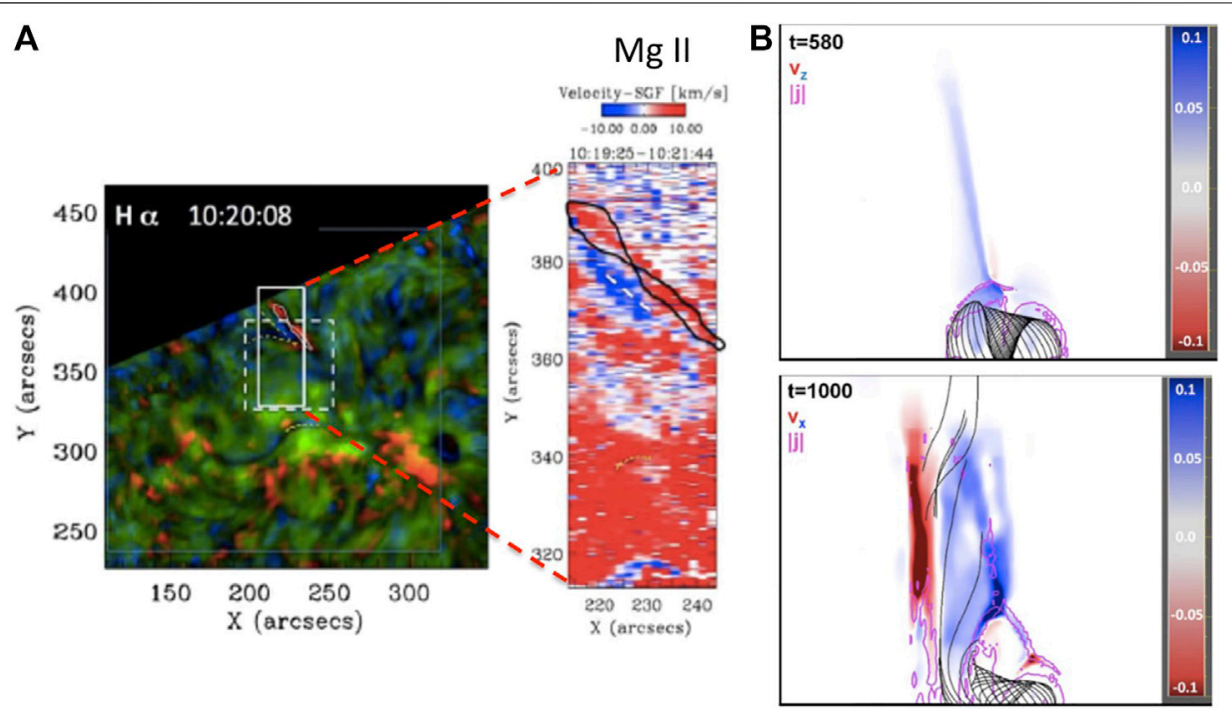

FIGURE 7 | Jet observation (A) in H $\alpha$ with the Meudon MSDP (left panel) in Mg II with the IRIS (middle panel) on 30 March 2017 : Dopplershift map (blue and red) combined with $\mathrm{H} \alpha$ intensity (yellow and green) and the related Dopplershift raster map in Mg II (from Ruan et al. (2019). (B) Dopplermaps obtained for two times in the 3D MHD simulation of a twisted jet showing that the opposite sides of the jet have opposite (red/blue) Dopplershifts [from Pariat et al. (2015)].

other studies (Panesar et al., 2016b). In some well-resolved, highcadence observations, the untwisting itself and helical structure were detected. Twisting has been observed in coronal hole jets too (e.g., Kumar et al. 2018).

Helical shape and twisting in jets have been further demonstrated by advanced spectroscopic methods. Using IRIS spectra, Doppler images revealed rotating jets showing blue and red shift on opposite sides of the jet axis (Jibben and Canfield, 2004; Cheung et al., 2015; Ruan et al., 2019). Magnetic reconnection of twisted flux tubes with their less twisted surroundings can account for the production and rotating motion of the jets. Cool jets or surges show also such blue/red Dopplershifts parallel to the structure (Figure 7A) (Tian et al., 2014; Ruan et al., 2019). Blue and red shift pattern is not always interpreted as a possible rotation (Schmieder et al., 1983; Tiwari et al., 2019), However, frequently, it is defined that the characteristics of the twisting jet is relatively common and does not depend on the temperature or the coronal environment. Twisting has been found in penumbra jets (Tiwari et al., 2018), and in active region jets (Lu et al., 2019; Joshi et al., 2021b) using Si IV, C II, and Mg II lines observed by the IRIS. The interpretation of the small jets is based on cartoons showing magnetic reconnection in mixed local magnetic field polarities. The spectra at the reconnection site show bidirectional flows either in the low chromosphere (Joshi et al., 2021b), in the corona (Ruan et al., 2019), or at the top of an emergent minifilament (Tiwari et al., 2019). Large Doppler flows can be found at the reconnection, for example, around $+/-100-200 \mathrm{~km} / \mathrm{s}$ (Joshi et al., 2021b). Bidirectional flows have also been interpreted as a signature of rotation in the jets themselves (Curdt et al., 2012; Pariat et al., 2016). However these Dopplershift flows are measured along the LoS which generally is nearly perpendicular to the direction of the observed jets. Therefore, they correspond to reconnection jets.

\subsection{MHD Models}

In the studies by Török et al. (2009) and Wyper and DeVore (2016), the helical jet consists of untwisting upflows driven by the propagation of torsional waves: these waves were induced by the sequential reconnection of twisted closed field lines with the straight open field. The global picture is due to multiple sequential reconnections. In numerical models of coronal jets generated in response to flux emergence, helical jets could be driven by untwisting upflows, for example, (Archontis and Hood, 2012).

In the study by Pariat et al. (2009), the helical jet is released by the interchange reconnection between open and closed magnetic fields, which generates a series of impulsive nonlinear Alfvénic or kink waves. This kind of torsional waves propagate with untwisting upflows along reconnection-formed open field lines and eject most of the twist (magnetic helicity) stored in the close domain (Figure 7B). In this model, the close domain possesses a given magnetic helicity with close twisted field lines, while in the emergence flux model, the flux emerges already twisted or the twist is created by untwisting upflows. In the study by Pariat et al. (2009), the twist is broadly distributed, driving reconnection at the breakout current sheet without the formation of the flux rope, while in the study by Wyper and DeVore (2016), the twist is concentrated along the PIL in a mini-filament which forms an eruptive flux rope. Multiple reconnection sites are possible below the FR and at the breakout current sheet, as was shown in coronal hole jets (Kumar et al., 2018). 
A

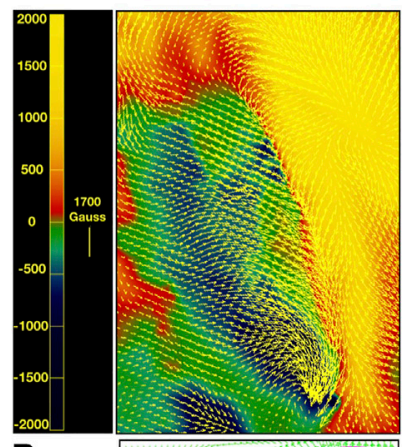

B
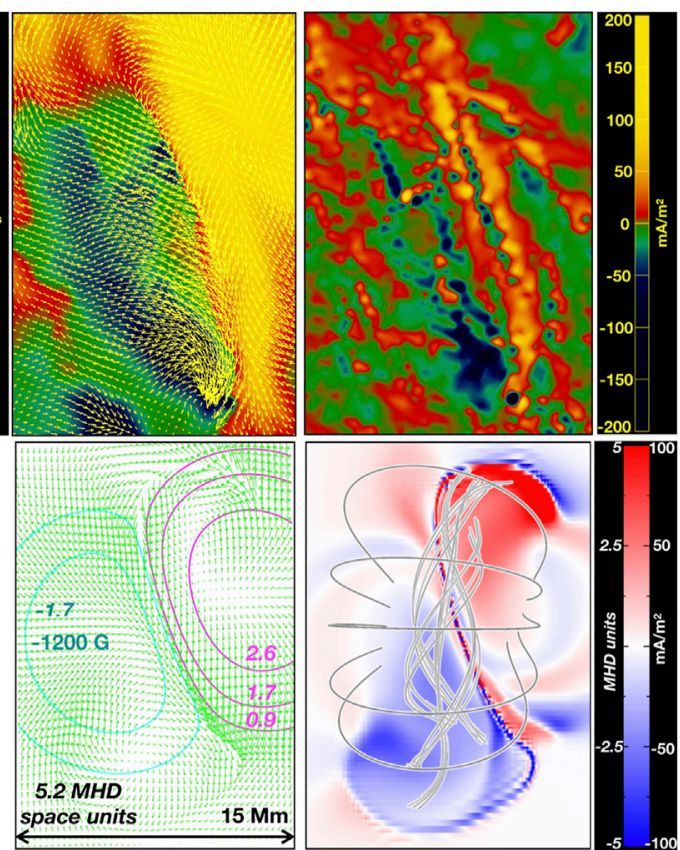

C

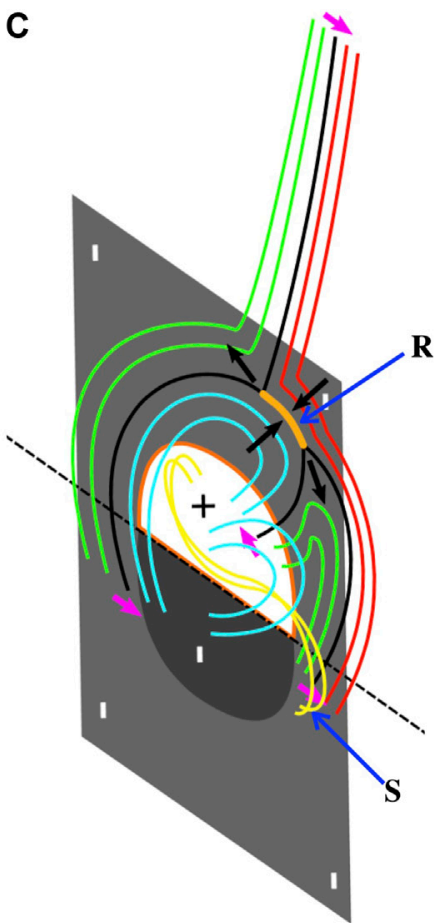

FIGURE 8 | Large flux rope detected in the HMI magnetic vector map computed with UNNOFIT code (Bommier, 2016) (A) Magnetic field Bz overlaid by arrows of the horizontal magnetic field (the yellow (dark) blue areas show the positive (negative) magnetic field polarity) and electric current density map Jz. (B) Comparison with the $\mathrm{OHM}$ MHD simulation of a flux rope. The vector pattern of observations and model looks the same as they are strongly nearly parallel to the PIL and converging together in the bottom part to the site of reconnection S (from Joshi et al. (2020b)). (C) Schematic view of magnetic field lines in the jet bald patch MHD simulation; in yellow is drawn the flux rope with a sigmoid shape [adapted from Wyper et al. (2019)]. The reconnection is at R in the study of Wyper et al. (2019) but, a reconnection at S will be a better fit to the Joshi et al. (2020b) observations.

\subsection{Transfer of Twist}

A new interesting case study was provided by a jet observed on 22 March 2019 with the SDO and IRIS studied by two groups using different tools (Joshi et al., 2020b; Yang et al., 2020). The active region was formed by a series of emerging flux, which evolved very rapidly and produced many micro flares. At the time of the jet, new emerging flux squeezed next to formerly emerged flux. The leading negative polarity of the bipole slipped along the following negative polarity of the older bipole (Joshi et al., 2020b). This scenario favors reconnection (Syntelis et al., 2015). At the time of the jet, a part of the two bipoles close to each other fragmented, and the jet occurred just at this smaller, newly created bipole. Therefore, the jet occurred between two arch filament systems which reconnected in a bald patch region. The bald patch region is transformed dynamically into a null point within a current sheet, and a twisted jet is expelled. This model of bald patch eruption has been studied by Wyper et al. (2019) (Figure 8 right panel). The question is: where does the energy that powers the jet and twist of the jet come from? The vector magnetograms unveil the existence of a large flux rope with sigmoid shape (Figure 8 left panels). Electric currents are detected in the hooks. The flux rope carries the energy release during the reconnection, and its twist is transferred during the reconnection. Joshi et al. (2020b) compared these observations to the results of a numerical simulation of the flux rope Aulanier et al. (2010); Zuccarello et al. (2015). The observed vector magnetic field vector pattern and the values of the vertical electric current density are in good agreement with synthetic vertical electric current density and vector B data from the MHD flux rope (FR) model, which reveals the FR location. The Mg II spectra observed at the base of the jet show a bidirectional extension with flows reaching $300 \mathrm{~km} / \mathrm{s}$. The spectra along the slit show a slow decrease of velocity along the slit as it crosses the main section of the jet, proving that the jet is rotating (Joshi et al., 2021b).

The second group explains this event differently (Yang et al., 2020). They performed a nonlinear force-free extrapolation and identified a null point, a fan and a long spine. This configuration differs from that inferred by Joshi et al. (2020b). They observed a small $\mathrm{H} \alpha$ filament with the high-resolution NVST telescope in the middle of the small bipole and suggested that this filament (FR) has a role in triggering the jet. They identified a second filament which does not correspond to the large FR found by the other group. As it was mentioned earlier, it is difficult to distinguish a filament from an arch filament system. This could be the case of this second filament which has, in fact, no role in their interpretation. They proposed a break-out model which might remove the overlaying arcades, leaving the small FR to erupt and turn into a blowout jet as in the scenario of the study by Sterling et al. (2015). This jet is explained by the breakout model for jets 
with mini-filament (Wyper et al., 2018). The conclusion of these two studies is that it is again difficult to understand the real driver of the jets and surges. In the study by Joshi et al. (2020b), the twist was transferred from a distant FR experimenting fragmentation, while in the study by Yang et al. (2020), the twist came directly from a mini-filament observed at the limit of the telescope resolution. Both interpretations are interesting. A data-driven study could help understand the evolution of the active region magnetic topology leading to this jet and other jets. On the theoretical point of view, data-driven simulations start to be very promising closer to the observations they may unveil the secret of jets (Guo et al., 2021).

\section{CONCLUSION AND PERSPECTIVE}

Solar jets have been observed for more than 50 years in multiwavelengths with steadily increasing spatial and temporal resolution instruments from ground-based and space telescopes. Jet characteristics (length, speed, and width) span large domains of ranges. They are observed all over the solar disk in active region, in coronal holes, and mainly at the edge of close structures neighboring open structures or large loops. Several recent partial or complete reviews exist on this topic (Innes et al., 2016; Pariat et al., 2016; Raouafi et al., 2016; Hinode Review Team, 2019; Shen, 2021; Schmieder et al., 2022).

In this study, we approach this subject with a critical view that differs from that of the previous reviews. Following a chronological order, we constantly present observations (SDO and IRIS) and the associated theoretical models, either with cartoons or 2D and 3D MHD simulations as they developed progressively. Substantial progress has been achieved concerning the analysis of the magnetic topology of jets. The results can be summarized as follows:

1. Magnetic reconnection triggers surges and jets and could occur in electric current layers associated with null point, bald patch, separatrices, and QSLs.

2. Convection is the main force which initiates photospheric motions leading to shear, flux cancellation, flux emergence, and consequently magnetic reconnection.

3. Electric current layers form between two different magnetic systems, for example, emerging magnetic flux and overlaying magnetic field. An intrusion of opposite polarity is in general detected in the magnetograms at the base of the jet. However, intrusions of opposite polarity are difficult to detect in quiet Sun and coronalhole magnetograms because the LoS fields are weak there and close to the HMI lower limit. DKIST should be more sensitive and might be able to demonstrate more conclusively whether flux cancellation is occurring.

4. Kinetic energy of reconnection jets comes from the dissipation of the magnetic field in the current sheet. The dissipation favors the changes of geometry of field lines, generating strong curvatures in the field lines. Therefore, a tension force operates, and the system grows. The jets have Alfvénic speed because the majority of the dissipated magnetic energy (and even 100\% in the Sweet Parker-type models) is converted into kinetic energy.

5. Twisted jets are frequently observed. Twisting should be already present in the closed region, either by kinking, flux rope formation, emergence of preexisting twisted flux, or postemergence rotation. The twist has to be transferred to the jethosting field lines through reconnection.

Kinetic energy from the untwisting jet comes from the reconnection between a twisted force-free field (fff) loop with an untwisted fff loop. The reconnected loop is twisted on one side and not on the other; it generates a non-fff at the interface, and therefore, the twist will be distributed by means of $\mathrm{J} \times \mathrm{B}$ along the field, a force which, therefore, also pushes the plasma in the direction of twisted field lines around and along the field lines. The speeds here depend on the magnitude of $\mathrm{J} \times \mathrm{B}$. so it may be different from Alfvenic speed.

Many questions about jets still stay open and need to be clarified. They are the seeds of many important questions relative to coronal heating (Berghmans et al., 2021; Panesar et al., 2021), sources of the solar wind (Fargette et al., 2021), acceleration of particles (Pick et al., 2006; Wang et al., 2006; Joshi et al., 2021a), and narrow coronal mass ejections (Shen et al., 2012; Panesar et al., 2016a; Kumar et al., 2021). Coordinated observations with new spacecraft [Parker Solar Probe - Fox et al. (2016) and Solar Orbiter- Müller et al. (2020)] and high ground-based instruments, for example, DKIST, EST will favor a breakthrough in our knowledge of solar jets and their related phenomena.

\section{AUTHOR CONTRIBUTIONS}

The author confirms being the sole contributor of this work and has approved it for publication.

\section{ACKNOWLEDGMENTS}

IRIS is a NASA small explorer mission developed and operated by LMSAL with mission operations executed at NASA Ames Research center and major contributions to downlink communications funded by the Norwegian Space Center (NSC, Norway) through an ESA PRODEX contract. We thank the SDO/AIA, SDO/HMI, and IRIS science teams for granting free access to the data. The author acknowledges the three referees who helped her improve the article substantially. BS thanks Reetika Joshi for achieving a few Figures. BS thanks Drs K.Shibata, S.Tiwari, A.Sterling, and Guillaume Aulanier for fruitful discussions on the theory of jets.

\section{SUPPLEMENTARY MATERIAL}

The Supplementary Material for this article can be found online at: https://www.frontiersin.org/articles/10.3389/fspas.2022.820183/ full\#supplementary-material 


\section{REFERENCES}

Alexander, D., and Fletcher, L. (1999). High-resolution Observations of Plasma Jets in the Solar Corona. Solar Phys. 190, 167-184. doi:10.1023/A:1005213826793

Archontis, V., and Hood, A. W. (2012). Magnetic Flux Emergence: a Precursor of Solar Plasma Expulsion. A\&A 537, A62. doi:10.1051/0004-6361/201116956

Archontis, V., Moreno-Insertis, F., Galsgaard, K., Hood, A., and O'Shea, E. (2004). Emergence of Magnetic Flux from the Convection Zone into the corona. A\&A 426, 1047-1063. doi:10.1051/0004-6361:20035934

Aulanier, G., Török, T., Démoulin, P., and DeLuca, E. E. (2010). Formation of Torus-Unstable Flux Ropes and Electric Currents in Erupting Sigmoids. ApJ 708, 314-333. doi:10.1088/0004-637X/708/1/314

Berghmans, D., Auchère, F., Long, D. M., Soubrié, E., Zhukov, A. N., Mierla, M., et al. (2021). Extreme UV Quiet Sun Brightenings Observed by Solar Orbiter/ EUI. arXiv e-prints , arXiv:2104.03382.

Bommier, V. (2016). Master Equation Theory Applied to the Redistribution of Polarized Radiation in the Weak Radiation Field Limit. AઐA 591, A60. doi:10. 1051/0004-6361/201526799

Canfield, R. C., Reardon, K. P., Leka, K. D., Shibata, K., Yokoyama, T., and Shimojo, M. (1996). H Alpha Surges and X-Ray Jets in AR 7260. ApJ 464, 1016. doi:10. $1086 / 177389$

Chandra, R., Mandrini, C. H., Schmieder, B., Joshi, B., Cristiani, G. D., Cremades, H., et al. (2017). Blowout Jets and Impulsive Eruptive Flares in a Bald-Patch Topology. AઐA 598, A41. doi:10.1051/0004-6361/201628984

Chen, H.-D., Zhang, J., and Ma, S.-L. (2012). The Kinematics of an Untwisting Solar Jet in a Polar Coronal Hole Observed bySDO/AIA. Res. Astron. Astrophys. 12, 573-583. doi:10.1088/1674-4527/12/5/009

Cheung, M. C. M., Pontieu, B. D., Tarbell, T. D., Fu, Y., Tian, H., Testa, P., et al. (2015). Homologous Helical Jets: Observations Byiris,sdo, Andhinodeand Magnetic Modeling with Data-Driven Simulations. ApJ 801, 83. doi:10.1088/ 0004-637X/801/2/83

Chiuderi-Drago, F., Mein, N., and Pick, M. (1986). Unstable Chromospheric Dark Features and Type-Iii Bursts Association. Sol. Phys. 103, 235-247. doi:10.1007/ BF00147827

Cirtain, J. W., Golub, L., Lundquist, L., van Ballegooijen, A., Savcheva, A., Shimojo, M., et al. (2007). Evidence for Alfve'n Waves in Solar X-ray Jets. Science 318, 1580-1582. doi:10.1126/science.1147050

Curdt, W., Tian, H., and Kamio, S. (2012). Explosive Events: Swirling Transition Region Jets. Sol. Phys. 280, 417-424. doi:10.1007/s11207-012-9940-9

De Pontieu, B., Polito, V., Hansteen, V., Testa, P., Reeves, K. K., Antolin, P., et al. (2021). A New View of the Solar Interface Region from the Interface Region Imaging Spectrograph (IRIS). Sol. Phys. 296, 84. doi:10.1007/s11207-02101826-0

De Pontieu, B., Title, A. M., Lemen, J. R., Kushner, G. D., Akin, D. J., Allard, B., et al. (2014). The Interface Region Imaging Spectrograph (IRIS). Solar Phys. 289, 2733-2779. doi:10.1007/s11207-014-0485-y

Démoulin, P., Héenoux, J. C., Priest, E. R., and Mandrini, C. H. (1996). QuasiSeparatrix Layers in Solar Flares. I. Method. Astron. Astrophys. 308, 643-655.

Fargette, N., Lavraud, B., Rouillard, A. P., Réville, V., Dudok De Wit, T., Froment, C., et al. (2021). Characteristic Scales of Magnetic Switchback Patches Near the Sun and Their Possible Association with Solar Supergranulation and Granulation. ApJ 919, 96. doi:10.3847/1538-4357/ac1112

Filippov, B. (1999). Observation of a 3d Magnetic Null Point in the Solar Corona. Solar Phys. 185, 297-309. doi:10.1023/A:1005124915577

Fox, N. J., Velli, M. C., Bale, S. D., Decker, R., Driesman, A., Howard, R. A., et al. (2016). The Solar Probe Plus Mission: Humanity's First Visit to Our Star. Space Sci. Rev. 204, 7-48. doi:10.1007/s11214-015-0211-6

Gu, X.-M., Lin, J., Li, K.-J., and Dun, J.-P. (1996). Multi-cloud Model (MCM) Method and its Applications to the Study on Asymmetric Spectral Profiles. Astrophys. Space Sci. 240, 263-271. doi:10.1007/BF00639591

Gudiksen, B. V., Carlsson, M., Hansteen, V. H., Hayek, W., Leenaarts, J., and Martínez-Sykora, J. (2011). The Stellar Atmosphere Simulation codeBifrost. AઐA 531, A154. doi:10.1051/0004-6361/201116520

Guo, Y., Démoulin, P., Schmieder, B., Ding, M. D., Vargas Domínguez, S., and Liu, Y. (2013). Recurrent Coronal Jets Induced by Repetitively Accumulated Electric Currents. AઐA 555, A19. doi:10.1051/0004-6361/201321229
Guo, Y., Zhong, Z., Ding, M. D., Chen, P. F., Xia, C., and Keppens, R. (2021). Dataconstrained Magnetohydrodynamic Simulation of a Long-Duration Eruptive Flare. ApJ 919, 39. doi:10.3847/1538-4357/ac10c8

He, J.-S., Marsch, E., Curdt, W., Tian, H., Tu, C.-Y., Xia, L.-D., et al. (2010). Magnetic and Spectroscopic Properties of Supergranular-Scale Coronal Jets and Erupting Loops in a Polar Coronal Hole. AઐA 519, A49. doi:10.1051/0004$6361 / 201014709$

He, J., Marsch, E., Tu, C., and Tian, H. (2009). Excitation of Kink Waves Due to Small-Scale Magnetic Reconnection in the Chromosphere. ApJ 705, L217-L222. doi:10.1088/0004-637X/705/2/L217

Hinode Review Team (2019). Achievements of Hinode in the First Eleven Years. Pub. Astron. Soc. Jpn. 71, R1. doi:10.1093/pasj/psz084

Hong, J.-C., Jiang, Y.-C., Yang, J.-Y., Zheng, R.-S., Bi, Y., Li, H.-D., et al. (2013). Twist in a Polar Blowout Jet. Res. Astron. Astrophys. 13, 253-258. doi:10.1088/ $1674-4527 / 13 / 3 / 001$

Innes, D. E., Bučík, R., Guo, L.-J., and Nitta, N. (2016). Observations of Solar X-ray and EUV Jets and Their Related Phenomena. Astron. Nachr. 337, 1024-1032. doi:10.1002/asna.201612428

Jibben, P., and Canfield, R. C. (2004). Twist Propagation in Ha Surges. ApJ 610, 1129-1135. doi: $10.1086 / 421727$

Joshi, R., Chandra, R., Schmieder, B., Moreno-Insertis, F., Aulanier, G., NóbregaSiverio, D., et al. (2020a). Case Study of Multi-Temperature Coronal Jets for Emerging Flux MHD Models. A\&A 639, A22. doi:10.1051/0004-6361/ 202037806

Joshi, R., Schmieder, B., Aulanier, G., Bommier, V., and Chandra, R. (2020b). The Role of Small-Scale Surface Motions in the Transfer of Twist to a Solar Jet from a Remote Stable Flux Rope. A\&A 642, A169. doi:10.1051/0004-6361/ 202038562

Joshi, R., Schmieder, B., Chandra, R., Aulanier, G., Zuccarello, F. P., and Uddin, W. (2017). Slippage of Jets Explained by the Magnetic Topology of NOAA Active Region 12035. Sol. Phys. 292, 152. doi:10.1007/s11207-017-1176-2

Joshi, R., Schmieder, B., Heinzel, P., Tomin, J., Chandra, R., and Vilmer, N. (2021a). Balmer Continuum Enhancement Detected in a Mini Flare Observed with IRIS. A $\sim A$ 654, A31. doi:10.1051/0004-6361/202141172

Joshi, R., Schmieder, B., Tei, A., Aulanier, G., Lörinčík, J., Chandra, R., et al. (2021b). Multi-thermal Atmosphere of a Mini-Solar Flare during Magnetic Reconnection Observed with IRIS. A\&A 645, A80. doi:10.1051/0004-6361/ 202039229

Joshi, R., Wang, Y., Chandra, R., Zhang, Q., Liu, L., and Li, X. (2020c). Cause and Kinematics of a Jetlike CME. ApJ 901, 94. doi:10.3847/1538-4357/abaf5a

Kumar, P., Karpen, J. T., Antiochos, S. K., Wyper, P. F., DeVore, C. R., and DeForest, C. E. (2018). Evidence for the Magnetic Breakout Model in an Equatorial Coronal-Hole Jet. ApJ 854, 155. doi:10.3847/1538-4357/aaab4f

Kumar, P., Karpen, J. T., Antiochos, S. K., Wyper, P. F., DeVore, C. R., and DeForest, C. E. (2019). Multiwavelength Study of Equatorial Coronal-Hole Jets. ApJ 873, 93. doi:10.3847/1538-4357/ab04af

Kumar, P., Karpen, J. T., Antiochos, S. K., Wyper, P. F., DeVore, C. R., and Lynch, B. J. (2021). From Pseudostreamer Jets to Coronal Mass Ejections: Observations of the Breakout Continuum. ApJ 907, 41. doi:10.3847/1538-4357/abca8b

Kumar, P., Yurchyshyn, V., Wang, H., and Cho, K.-S. (2015). Formation and Eruption of a Small Flux Rope in the Chromosphere Observed by Nst,iris, Andsdo. ApJ 809, 83. doi:10.1088/0004-637X/809/1/83

Kundu, M. R., Raulin, J. P., Nitta, N., Hudson, H. S., Shimojo, M., Shibata, K., et al. (1995). Detection of Nonthermal Radio Emission from Coronal X-Ray Jets. Astrophys. J. Lett. 447, L135. doi:10.1086/309567

Lemen, J. R., Title, A. M., Akin, D. J., Boerner, P. F., Chou, C., Drake, J. F., et al. (2012). The Atmospheric Imaging Assembly (AIA) on the Solar Dynamics Observatory (SDO). Sol. Phys. 275, 17-40. doi:10.1007/s11207-011-9776-8

Liu, Z., Xu, J., Gu, B.-Z., Wang, S., You, J.-Q., Shen, L.-X., et al. (2014). New Vacuum Solar Telescope and Observations with High Resolution. Res. Astron. Astrophys. 14, 705-718. doi:10.1088/1674-4527/14/6/009

Lu, L., Feng, L., Li, Y., Li, D., Ning, Z., and Gan, W. (2019). Spectroscopic and Stereoscopic Observations of the Solar Jets. ApJ 887, 154. doi:10.3847/1538$4357 / \mathrm{ab} 530 \mathrm{c}$

Madjarska, M. S. (2011). Dynamics and Plasma Properties of an X-ray Jet from SUMER, EIS, XRT, and EUVI A \& B Simultaneous Observations. AઐA 526, A19. doi:10.1051/0004-6361/201015269 
McGlasson, R. A., Panesar, N. K., Sterling, A. C., and Moore, R. L. (2019). Magnetic Flux Cancellation as the Trigger Mechanism of Solar Coronal Jets. ApJ 882, 16. doi:10.3847/1538-4357/ab2fe3

Mein, N., Mein, P., Heinzel, P., Vial, J. C., Malherbe, J. M., and Staiger, J. (1996). Cloud Model with Variable Source Function for Solar $\mathrm{H} \alpha$ Structures. Astron. Astrophys. 309, 275-283.

Mein, P. (1977). Multi-channel Subtractive Spectrograph and Filament Observations. Sol. Phys. 54, 45-51. doi:10.1007/BF00146423

Moore, R. L., Cirtain, J. W., Sterling, A. C., and Falconer, D. A. (2010). Dichotomy of Solar Coronal Jets: Standard Jets and Blowout Jets. ApJ 720, 757-770. doi:10. 1088/0004-637X/720/1/757

Moore, R. L., Sterling, A. C., Falconer, D. A., and Robe, D. (2013). The Cool Component and the Dichotomy, Lateral Expansion, and Axial Rotation of Solar X-Ray Jets. ApJ 769, 134. doi:10.1088/0004-637X/769/2/134

Moreno-Insertis, F., and Galsgaard, K. (2013). Plasma Jets and Eruptions in Solar Coronal Holes: A Three-Dimensional Flux Emergence Experiment. ApJ 771, 20. doi:10.1088/0004-637X/771/1/20

Moreno-Insertis, F., Galsgaard, K., and Ugarte-Urra, I. (2008). Jets in Coronal Holes: Hinode Observations and Three-Dimensional Computer Modeling. ApJ 673, L211-L214. doi:10.1086/527560

Muglach, K. (2021). The Photospheric Footpoints of Solar Coronal Hole Jets. ApJ 909, 133. doi:10.3847/1538-4357/abd5ad

Mulay, S. (2018). EUV Spectroscopy of Solar Active Region Jets (UK: Department of Applied Mathematics and Theoretical Physics, University of Cambridge). Ph.D. thesis. An optional note. doi:10.17863/CAM.25040

Mulay, S. M., Sharma, R., Valori, G., Vásquez, A. M., Del Zanna, G., Mason, H., et al. (2019). Study of the Spatial Association between an Active Region Jet and a Nonthermal Type III Radio Burst. AઐA 632, A108. doi:10.1051/0004-6361/201936369

Müller, D., St. Cyr, O. C. O. C., Zouganelis, I., Gilbert, H. R., Marsden, R., NievesChinchilla, T., et al. (2020). The Solar Orbiter mission. A\&aastron. Astrophys. 642, A1. doi:10.1051/0004-6361/202038467

Musset, S., Jeunon, M., and Glesener, L. (2020). Statistical Study of Hard X-Ray Emitting Electrons Associated with Flare-Related Coronal Jets. ApJ 889, 183. doi:10.3847/1538-4357/ab6222

Ni, L., Chen, Y., Peter, H., Tian, H., and Lin, J. (2021). A Magnetic Reconnection Model for Hot Explosions in the Cool Atmosphere of the Sun. AઐA 646, A88. doi:10.1051/0004-6361/202039239

Nisticò, G., Bothmer, V., Patsourakos, S., and Zimbardo, G. (2009). Characteristics of EUV Coronal Jets Observed with STEREO/SECCHI. Sol. Phys. 259, 87-108. doi:10.1007/s11207-009-9424-8

Nóbrega-Siverio, D., Martínez-Sykora, J., Moreno-Insertis, F., and Voort, L. R. v. d. (2017). Surges and Si Iv Bursts in the Solar Atmosphere: UnderstandingIRISand SST Observations through RMHD Experiments. ApJ 850, 153. doi:10.3847/ 1538-4357/aa956c

Nóbrega-Siverio, D., Moreno-Insertis, F., and Marti'nez-Sykora, J. (2016). The Cool Surge Following Flux Emergence in a Radiation-MHD Experiment. ApJ 822, 18. doi:10.3847/0004-637X/822/1/18

Panesar, N. K., Moore, R. L., and Sterling, A. C. (2020). Onset of Magnetic Explosion in Solar Coronal Jets in Quiet Regions on the Central Disk. ApJ 894, 104. doi:10.3847/1538-4357/ab88ce

Panesar, N. K., Sterling, A. C., Moore, R. L., and Chakrapani, P. (2016b). Magnetic Flux Cancelation as the Trigger of Solar Quiet-Region Coronal Jets. ApJ 832, L7. doi:10.3847/2041-8205/832/1/L7

Panesar, N. K., Sterling, A. C., and Moore, R. L. (2016a). Homologous Jet-Driven Coronal Mass Ejections from Solar Active Region 12192. ApJ 822, L23. doi:10. 3847/2041-8205/822/2/L23

Panesar, N. K., Sterling, A. C., and Moore, R. L. (2018a). Magnetic Flux Cancelation as the Trigger of Solar Coronal Jets in Coronal Holes. ApJ 853, 189. doi:10.3847/ 1538-4357/aaa3e9

Panesar, N. K., Sterling, A. C., and Moore, R. L. (2017). Magnetic Flux Cancellation as the Origin of Solar Quiet-Region Pre-jet Minifilaments. ApJ 844, 131. doi:10. 3847/1538-4357/aa7b77

Panesar, N. K., Sterling, A. C., Moore, R. L., Tiwari, S. K., De Pontieu, B., and Norton, A. A. (2018b). IRIS and SDO Observations of Solar Jetlets Resulting from Network-Edge Flux Cancelation. ApJ 868, L27. doi:10.3847/2041-8213/aaef37

Panesar, N. K., Tiwari, S. K., Berghmans, D., Cheung, M. C. M., Müller, D., Auchere, F., et al. (2021). The Magnetic Origin of Solar Campfires. ApJL 921, L20. doi:10.3847/2041-8213/ac3007
Pariat, E., Antiochos, S. K., and DeVore, C. R. (2009). A Model for Solar Polar Jets. ApJ 691, 61-74. doi:10.1088/0004-637X/691/1/61

Pariat, E., Dalmasse, K., DeVore, C. R., Antiochos, S. K., and Karpen, J. T. (2016). A Model for Straight and Helical Solar Jets. AઐA 596, A36. doi:10.1051/00046361/201629109

Pariat, E., Dalmasse, K., DeVore, C. R., Antiochos, S. K., and Karpen, J. T. (2015). Model for Straight and Helical Solar Jets. A\&A 573, A130. doi:10.1051/00046361/201424209

Patsourakos, S., Pariat, E., Vourlidas, A., Antiochos, S. K., and Wuelser, J. P. (2008). STEREO SECCHI Stereoscopic Observations Constraining the Initiation of Polar Coronal Jets. ApJ 680, L73-L76. doi:10.1086/589769

Pick, M., Mason, G. M., Wang, Y. M., Tan, C., and Wang, L. (2006). Solar Source Regions for3He-rich Solar Energetic Particle Events Identified Using Imaging Radio, Optical, and Energetic Particle Observations. ApJ 648, 1247-1255. doi: $10.1086 / 505926$

Priest, E. R., Parnell, C. E., and Martin, S. F. (1994). A Converging Flux Model of an $\mathrm{X}$-Ray Bright Point and an Associated Canceling Magnetic Feature. ApJ 427, 459. doi:10.1086/174157

Raouafi, N. E., Patsourakos, S., Pariat, E., Young, P. R., Sterling, A. C., Savcheva, A., et al. (2016). Solar Coronal Jets: Observations, Theory, and Modeling. Space Sci. Rev. 201, 1-53. doi:10.1007/s11214-016-0260-5

Roy, J. R. (1973). The Magnetic Properties of Solar Surges. Sol. Phys. 28, 95-114. doi:10.1007/BF00152915

Ruan, G., Schmieder, B., Masson, S., Mein, P., Mein, N., Aulanier, G., et al. (2019). Bidirectional Reconnection Outflows in an Active Region. ApJ 883, 52. doi:10. 3847/1538-4357/ab3657

Savcheva, A., Cirtain, J., Deluca, E. E., Lundquist, L. L., Golub, L., Weber, M., et al. (2007). A Study of Polar Jet Parameters Based on Hinode XRT Observations. Publ. Astron. Soc. Jpn. 59, S771-S778. doi:10.1093/pasj/59.sp3.S771

Scherrer, P. H., Schou, J., Bush, R. I., Kosovichev, A. G., Bogart, R. S., Hoeksema, J. T., et al. (2012). The Helioseismic and Magnetic Imager (HMI) Investigation for the Solar Dynamics Observatory (SDO). Sol. Phys. 275, 207-227. doi:10. 1007/s11207-011-9834-2

Schmieder, B., Archontis, V., and Pariat, E. (2014). Magnetic Flux Emergence along the Solar Cycle. Space Sci. Rev. 186, 227-250. doi:10.1007/s11214-014-0088-9

Schmieder, B., Golub, L., and Antiochos, S. K. (1994). Comparison between Cool and Hot Plasma Behaviors of Surges. ApJ 425, 326-330. doi:10.1086/173987

Schmieder, B., Guo, Y., Moreno-Insertis, F., Aulanier, G., Yelles Chaouche, L., Nishizuka, N., et al. (2013). Twisting Solar Coronal Jet Launched at the Boundary of an Active Region. AઐA 559, A1. doi:10.1051/0004-6361/ 201322181

Schmieder, B., Joshi, R., and Chandra, R. (2022). "Solar Jets Observed with the Interface Region Imaging Spectrograph (IRIS)," in Adv. Space R., 0, A1. doi:10. 1016/j.asr.2021.12.013

Schmieder, B., Mein, N., Shibata, K., van Driel-Gesztelyi, L., and Kurokawa, H. (1996a). Chromospheric Ejections and Their Signatures in X-ray Observed by YOHKOH. Adv. Space Res. 17, 193-196. doi:10.1016/0273-1177(95)00566-W

Schmieder, B., Mein, P., Martres, M. J., and Tandberg-Hanssen, E. (1984). Dynamic Evolution of Recurrent Mass Ejections Observed in H? and Civ Lines. Sol. Phys. 94, 133-150. doi:10.1007/BF00154814

Schmieder, B., Mein, P., Simnett, G. M., and Tandberg-Hanssen, E. (1988). An Example of the Association of X-ray and UV Emission with H-Alpha Surges. Astron. Astrophys. 201, 327-338.

Schmieder, B., Mein, P., Vial, J. C., and Tandberg-Hanssen, E. (1982). A Surge Obsrved in $\mathrm{Ha}$ and CIV. Adv. Space Res. 2, 225-228. doi:10.1016/0273$1177(82) 90203-4$

Schmieder, B., Mein, P., Vial, J. C., and Tandberg-Hanssen, E. (1983). Dynamics of a Surge Observed in the C IV and H Alpha Lines. Astron. Astrophys. 127, 337-344.

Schmieder, B., Rovira, M., Simnett, G. M., Fontenla, J. M., and Tandberg-Hanssen, E. (1996b). Subflares and Surges in AR 2744 during the Solar Maximum Mission. Astron. Astrophys. 308, 957-969.

Schmieder, B., Shibata, K., van Driel-Gesztelyi, L., and Freeland, S. (1995). Ha Surges and Associated Soft X-ray Loops. Sol. Phys. 156, 245-264. doi:10.1007/ BF00670226

Schmieder, B., van Driel-Gesztelyi, L., Gerlei, O., and Simnett, G. M. (1993). A Study of Surges and Flares within an Active Region. Sol. Phys. 146, 163-176. doi:10.1007/BF00662177 
Shen, Y., Liu, Y. D., Su, J., Qu, Z., and Tian, Z. (2017). On a Solar Blowout Jet: Driving Mechanism and the Formation of Cool and Hot Components. ApJ 851, 67. doi:10.3847/1538-4357/aa9a48

Shen, Y., Liu, Y., Su, J., and Deng, Y. (2012). On a Coronal Blowout Jet: The First Observation of a Simultaneously Produced Bubble-like CME and a Jet-like CME in a Solar Event. ApJ 745, 164. doi:10.1088/0004-637X/745/2/164

Shen, Y. (2021). Observation and Modelling of Solar Jets. Proc. R. Soc. A. 477, 20200217. doi:10.1098/rspa.2020.0217

Shibata, K. (1998). A Unified Model of Solar Flares. Dordrecht: Springer Netherlands, 187-196. doi:10.1007/978-94-011-5220-4_32

Shibata, K. (1999). Evidence of Magnetic Reconnection in Solar Flares and a Unified Model of Flares. Astrophys. Space Sci. 264, 129-144. doi:10.1007/97894-011-4203-8_11

Shibata, K., Ishido, Y., Acton, L. W., Strong, K. T., Hirayama, T., Uchida, Y., et al. (1992). Observations of X-Ray Jets with the YOHKOH Soft X-Ray Telescope. Pub. Astron. Soc. Jpn. 44, L173-L179.

Shibata, K., Nitta, N., Strong, K. T., Matsumoto, R., Yokoyama, T., Hirayama, T., et al. (1994). A Gigantic Coronal Jet Ejected from a Compact Active Region in a Coronal Hole. ApJ 431, L51-L53. doi:10.1086/187470

Shibata, K., and Uchida, Y. (1985). A Magnetodynamic Mechanism for the Formation of Astrophysical Jets. I - Dynamical Effects of the Relaxation of Nonlinear Magnetic Twists. Pub. Astron. Soc. Jpn. 37, 31-46.

Shibata, K., and Uchida, Y. (1986). Sweeping-magnetic-twist Mechanism for the Acceleration of Jets in the Solar Atmosphere. Sol. Phys. 103, 299-310. doi:10. 1007/BF00147831

Shibata, K., Yokoyama, T., and Shimojo, M. (1996). Coronal X-ray Jets Observed with Yohkoh/SXT. Adv. Space Res. 17, 197-200. doi:10.1016/0273-1177(95) 00567-X

Shimojo, M., Shibata, K., Yokoyama, T., and Hori, K. (2001). One-dimensional and Pseudo-two-dimensional Hydrodynamic Simulations of Solar X-Ray Jets. ApJ 550, 1051-1063. doi:10.1086/319788

Sterling, A. C., Harra, L. K., Moore, R. L., and Falconer, D. A. (2019). A Two-Sided Loop X-Ray Solar Coronal Jet Driven by a Minifilament Eruption. ApJ 871, 220. doi:10.3847/1538-4357/aaf1d3

Sterling, A. C., and Moore, R. L. (2020). Coronal-jet-producing Minifilament Eruptions as a Possible Source of Parker Solar Probe Switchbacks. ApJ 896, L18. doi:10.3847/2041-8213/ab96be

Sterling, A. C., Moore, R. L., Falconer, D. A., and Adams, M. (2015). Small-scale Filament Eruptions as the Driver of X-ray Jets in Solar Coronal Holes. Nature 523, 437-440. doi:10.1038/nature14556

Sterling, A. C., Moore, R. L., and Panesar, N. K. (2018). Magnetic Flux Cancelation as the Buildup and Trigger Mechanism for CME-Producing Eruptions in Two Small Active Regions. ApJ 864, 68. doi:10.3847/1538-4357/aad550

Sterling, A. C., Shibata, K., and Mariska, J. T. (1994). Jets and Brightenings Generated by Energy Deposition in the Middle and Upper Solar Chromosphere. Space Sci. Rev. 70, 77-80. doi:10.1007/BF00777845

Syntelis, P., Archontis, V., Gontikakis, C., and Tsinganos, K. (2015). Emergence of Non-twisted Magnetic fields in the Sun: Jets and Atmospheric Response. AઐA 584, A10. doi:10.1051/0004-6361/201423781

Tandberg-Hanssen, E. (1974). Solar Prominences, Vol. 12. Dordrecht, Netherlands: D.Reidl Publ.Co

Tian, H., DeLuca, E. E., Cranmer, S. R., De Pontieu, B., Peter, H., Martínez-Sykora, J., et al. (2014). Prevalence of Small-Scale Jets from the Networks of the Solar Transition Region and Chromosphere. Science 346, 1255711. doi:10.1126/ science. 1255711

Tiwari, S. K., Moore, R. L., De Pontieu, B., Tarbell, T. D., Panesar, N. K., Winebarger, A. R., et al. (2018). Evidence of Twisting and Mixed-Polarity
Solar Photospheric Magnetic Field in Large Penumbral Jets: IRIS and Hinode Observations. ApJ 869, 147. doi:10.3847/1538-4357/aaf1b8

Tiwari, S. K., Panesar, N. K., Moore, R. L., Pontieu, B. D., Winebarger, A. R., Golub, L., et al. (2019). Fine-scale Explosive Energy Release at Sites of Prospective Magnetic Flux Cancellation in the Core of the Solar Active Region Observed by Hi-C 2.1, IRIS, and SDO. ApJ 887, 56. doi:10.3847/1538-4357/ab54c1

Török, T., Aulanier, G., Schmieder, B., Reeves, K. K., and Golub, L. (2009). FanSpine Topology Formation through Two-step Reconnection Driven by Twisted Flux Emergence. ApJ 704, 485-495. doi:10.1088/0004-637X/704/1/485

Tsuneta, S., Acton, L., Bruner, M., Lemen, J., Brown, W., Caravalho, R., et al. (1991). The Soft X-ray Telescope for the SOLAR-A mission. Sol. Phys. 136, 37-67. doi:10.1007/BF00151694

Wang, Y. M., Pick, M., and Mason, G. M. (2006). Coronal Holes, Jets, and the Origin of3He-rich Particle Events. ApJ 639, 495-509. doi:10.1086/499355

Woodgate, B. E., Tandberg-Hanssen, E. A., Bruner, E. C., Beckers, J. M., Brandt, J. C., Henze, W., et al. (1980). The Ultraviolet Spectrometer and Polarimeter on the Solar Maximum Mission. Sol. Phys. 65, 73-90. doi:10.1007/BF00151385

Wyper, P. F., DeVore, C. R., and Antiochos, S. K. (2018). A Breakout Model for Solar Coronal Jets with Filaments. ApJ 852, 98. doi:10.3847/1538-4357/aa9ffc

Wyper, P. F., DeVore, C. R., and Antiochos, S. K. (2019). Numerical Simulation of Helical Jets at Active Region Peripheries. Mon. Not. Roy. Astron. Soc. 490, 3679-3690. doi:10.1093/mnras/stz2674

Wyper, P. F., and DeVore, C. R. (2016). Simulations of Solar Jets Confined by Coronal Loops. ApJ 820, 77. doi:10.3847/0004-637X/820/1/77

Yang, L., Peter, H., He, J., Tu, C., Wang, L., Zhang, L., et al. (2018). Formation of Cool and Warm Jets by Magnetic Flux Emerging from the Solar Chromosphere to Transition Region. ApJ 852, 16. doi:10.3847/1538-4357/aa9996

Yang, S., Zhang, Q., Xu, Z., Zhang, J., Zhong, Z., and Guo, Y. (2020). Imaging and Spectral Study on the Null Point of a Fan-Spine Structure during a Solar Flare. ApJ 898, 101. doi:10.3847/1538-4357/ab9ac7

Yokoyama, T., and Shibata, K. (1995). Magnetic Reconnection as the Origin of X-ray Jets and Ha Surges on the Sun. Nature 375, 42-44. doi:10.1038/375042a0

Yokoyama, T., and Shibata, K. (1996). Numerical Simulation of Solar Coronal X-Ray Jets Based on the Magnetic Reconnection Model. Publ. Astron. Soc. Jpn. 48, 353-376. doi:10.1093/pasj/48.2.353

Young, P. R., and Muglach, K. (2014). Solar Dynamics Observatory and Hinode Observations of a Blowout Jet in a Coronal Hole. Sol. Phys. 289, 3313-3329. doi:10.1007/s11207-014-0484-z

Zuccarello, F. P., Aulanier, G., and Gilchrist, S. A. (2015). Critical Decay Index at the Onset of Solar Eruptions. ApJ 814, 126. doi:10.1088/0004-637X/814/2/126

Conflict of Interest: The author declares that the research was conducted in the absence of any commercial or financial relationships that could be construed as a potential conflict of interest.

Publisher's Note: All claims expressed in this article are solely those of the authors and do not necessarily represent those of their affiliated organizations, or those of the publisher, the editors, and the reviewers. Any product that may be evaluated in this article, or claim that may be made by its manufacturer, is not guaranteed or endorsed by the publisher.

Copyright () 2022 Schmieder. This is an open-access article distributed under the terms of the Creative Commons Attribution License (CC BY). The use, distribution or reproduction in other forums is permitted, provided the original author $(s)$ and the copyright owner(s) are credited and that the original publication in this journal is cited, in accordance with accepted academic practice. No use, distribution or reproduction is permitted which does not comply with these terms. 